\title{
Random Fields as Solutions of the Inhomogeneous Quaternionic Cauchy-Riemann Equation. I. Invariance and Analytic Continuation
}

\author{
S. Albeverio ${ }^{1,3}, \mathrm{~K}$. Iwata ${ }^{1}$, and T. Kolsrud ${ }^{2}$ \\ 1 Ruhr-Universität Bochum and SFB 237, Bochum-Essen-Düsseldorf, \\ Federal Republic of Germany \\ ${ }^{2}$ Kungliga Tekniska Högskolan, Stockholm, Sweden \\ 3 BiBo-S Research Centre, Universität Bielefeld, Federal Republic of Germany, \\ and CERFIM, Locarno, Switzerland
}

\begin{abstract}
We consider random fields $A$ satisfying the quaternionic CauchyRiemann equation $\partial A=F$, where $F$ is white noise. Under appropriate conditions on $F, A$ is invariant under the proper Euclidean group in four dimensions, but in general not under time reflection. The Schwinger functions can be analytically continued to Wightman functions satisfying the relativistic postulates on invariance, spectral property and locality.
\end{abstract}

\section{Introduction}

In recent years models of local interacting relativistic quantum fields of scalar, vector, or gauge type have been constructed (see e.g. $[2,6,7,20,28,34]$ ) in space-time dimensions $d<4$. Since the basic work of Nelson's, the construction of such fields has been closely connected with the construction of Euclidean (i.e. invariant in law under the Euclidean group) random fields over $\mathbf{R}^{d}$ having suitable "Markovian" properties. ${ }^{1}$ The case $d=4$ however, has remained open, from the quantum field as well as the Euclidean Markov field point of view. ${ }^{2}$

The present article is the first one in a planned series where we investigate random fields and quantum fields over $\mathbf{R}^{4}$. Our 4-component random fields $A$ are obtained by solving a first order elliptic partial differential equation

$$
\partial A=F,
$$

where $F$ is a 4-component Gauss-Poisson white noise with specific Euclidean invariance. We shall formulate the above by making use of ample algebraic structure of the field $\mathbf{H}$ of quaternions, which is isomorphic to $\mathbf{R}^{4}$ as vector spaces over the reals.

Some results have already been announced in [0] and [3-5]. Reference [12] contains a correction to [5] as well as further discussions and proofs.

${ }^{1}$ For $d=2$ most scalar models have been shown to fulfill the (strict) global Markov property (in particular with respect to half-spaces) and Nelson's axioms. See [13] and references therein

${ }^{2}$ For some partial results, see e.g. $[0,1,6-9,14,18]$ and references therein 
In the present paper we resume the discussion, extending it in particular towards the study of analytic continuation to Minkowski space-time of the moments (Schwinger functions) of the Euclidean random field $A$ solving (1.1). Before going into more details, let us stress the basic ideas behind the construction, making in fact the model fairly uniquely tied to the 4-dimensionality of the underlying space-time.

A stochastic equation of the form $L A=F$, with $L$ elliptic with constant coefficients, and $F$ a Euclidean white noise, looks, due to its covariance properties, a promising candidate for constructing Euclidean Markov random fields. If $L$ is also of first order, we expect the resulting random field to possess the sharp Markov property. Furthermore, covariance properties force us to take $L$ purely first-order, i.e. no constant term is allowed. The well-known group isomorphism, again special for dimension $4, S O(4) \cong(S U(2) \times S U(2)) / \mathbf{Z}_{2}$ (see [15], for instance) is crucial. Using the isomorphisms between $\mathbb{R}^{4}$ and $\mathbf{H}$, and $S U(2)$ with the group $\mathrm{Sp}$ (1) of quaternions of norm 1, we arrive at Eq. (1.1), as discussed below.

That we take $F$ to be a white noise, i.e. a translation invariant random field with independent values at different points in the sense of [19], is motivated by our desire to get Markovian fields. Let us remark here that when $F$ is Gaussian, the corresponding random field $A$ is a realisation of the free electromagnetic Euclidean potential field. In the case when $F$ is non-Gaussian, $A$ itself is non-Gaussian and can be looked upon as a mathematical realisation of models for interacting electromagnetic non-linear quantum field theories. A forerunner and prototype in this respect is the Born-Infeld model ([17]).

In a series of articles [2,7-11], we have investigated various group-valued random fields as realisations or extensions of(non-abelian) gauge fields. The present work is closely related to the previous articles, thinking of $\mathbf{H}$ as the Lie algebra of $U(2){ }^{3}$

An important, and, we think, attractive feature of our models is that they are in a genuine sense explicit. To mention but one example, we achieve the analytic continuation of the Schwinger functions directly "by hand," without involving much of the axiomatic frameworks. We look at our model as a, at least partial, realisation of the goal of uniting - in form of a local quantum field theory over 4-dimensional space-time - the ideas of quantum theory and special relativity in a well-defined mathematical setting.

This article is devoted to invariance properties and existence of the Wightman functions of the models, as analytic continuation of the Schwinger functions. We show that our model possesses the basic invariance, spectrum and locality, which are required in axiomatic approaches of relativistic quantum fields. As to other properties, including the positivity of Wightman functions (in which one encounters problems related to those of gauge field theories), we plan to discuss them in forthcoming work, where we shall also discuss Markov, scaling and cluster properties, together with taking a closer look at the regularity of the Wightman functions.

In Sect. 2 the representation theory necessary for studying invariance properties is presented. In Sect. 3 we construct random fields which are invariant

${ }^{3}$ For the study of stochastic equations associated with lower dimensional Euclidean random fields, see [2, 21-24, 28, 37, 38], and references therein. See also [32] 
under rotations and translations, i.e. under the semi-direct product $(\operatorname{Sp}(1)$ $\times \mathrm{Sp}(1)) \odot H$, but in general not under reflections (cf. [12]).

The fourth and final section is devoted to analytic continuation. The set-up is carefully tailored to suit an extension to a more general geometric framework (fields over manifolds). We show that the Schwinger functions can be analytically continued to Wightman functions (Theorem 4.21), which satisfy the postulates on invariance, spectral property and locality.

\section{Quaternions, Representations, and Invariance}

A possible non-commutative generalisation of the theory of complex-analytic functions may be directed to functions with values in Clifford modules. However, due to the lack of commutativity, the basic result which holds in the complex valued case fails, viz. the three definitions in terms of complex differential, CauchyRiemann equation and power series expansion no longer agree (see e.g. Sudbery [36]). The Cauchy-Riemann equation remains a natural candidate among others. We shall make use of the quaternionic inhomogeneous Cauchy-Riemann equation to construct random fields, for the following reasons. Since the Cauchy-Riemann operator is elliptic and of first order, if the driving term is white noise, say, we expect the resulting random field to possess the sharp Markov property. Moreover the dimensionality of the physical space-time entails the employment of quaternions on us.

Let $\mathbf{H}$ be the (skew-) field of quaternionic numbers, which is algebraically characterized as the unique associative division algebra over $\mathbf{R}$ of dimension four. We denote the multiplicative identity by 1 . There is a distinct anti-automorphism of $\mathbf{H}$, written

$$
\mathbf{H} \ni x \mapsto \bar{x} \in \mathbf{H},
$$

and called conjugation, which is uniquely determined by property

$$
x \bar{x} \in Z(\mathbf{H}) \quad \forall x \in \mathbf{H},
$$

where $Z$ denotes centre.

By the triviality of $Z(\mathbf{H})$ and the division property we have

$$
x \bar{x}=\bar{x} x=|x|^{2} 1, \quad x \in \mathbf{H}
$$

for some non-negative quantity $|x|$. Therefore $\overline{1}=1$ implies involutivity: $\overline{\bar{x}}=x$. Observing that

$$
x \bar{y}+y \bar{x}=\bar{x} y+\bar{y} x=\frac{1}{2}\left(|x+y|^{2}-|x-y|^{2}\right) 1, \quad x, y \in \mathbf{H},
$$

we deduce that $|\cdot|$ is a Euclidean norm. The associated inner product will be denoted by $(\cdot, \cdot)$. It is also seen that

$$
|x y|=|x||y|, \quad|\bar{x}|=|x| .
$$

Imitating the notations in the case of $\mathbf{C}$, we write $\operatorname{Re} x \equiv x^{0}=\frac{1}{2}(x+\bar{x})$, and $\operatorname{Im} x \equiv \vec{x}$ $=\frac{1}{2}(x-\bar{x})$. Choose two elements $i$ and $j$ satisfying the orthogonality condition

$$
(1, i)=(1, j)=0, \quad(i, j)=0, \quad \text { and } \quad|i|=|j|=1 .
$$


Equivalently

$$
\bar{i}=-i, \quad \bar{j}=-j, \quad i j+j i=0, \quad \text { and } \quad i^{2}=j^{2}=-1 .
$$

These relations display the isomorphism between $\mathbf{H}$ and the Clifford algebra over $\mathbf{R}^{2}$, and we see that $\{1, i, j, i j\}$ forms an orthonormal basis. Writing $k=i j$, we obtain the familiar multiplication rules

$$
i^{2}=j^{2}=k^{2}=-1, \quad i j=-j i=k, \quad j k=-k j=i, \quad k i=-i k=j .
$$

Since the orientation $\{1, i, j, i j\}$ is independent of the choice of the pair $(i, j)$, we may speak of the canonical orientation of $\mathbf{H}$.

Let $E_{4}$ be a four-dimensional Euclidean space. We choose $\mathbf{H}$ as the difference space for $E_{4}$, viz. the additive group $\mathrm{H}$ acts transitively and freely on $E_{4}$ from the right. Using a basis $\{1, i, j, k\}$ of $\mathbf{H}$ and an origin 0 in $E_{4}$, we introduce a coordinate system $\left\{x^{0}, x^{1}, x^{2}, x^{3}\right\}$ in $E_{4}$ :

$$
p=0+\left(x^{0} 1+x^{1} i+x^{2} j+x^{3} k\right), \quad p \in E_{4} .
$$

We can now define the Cauchy-Riemann operator. Regard the algebra $\mathbf{H}$ as a left $\mathbf{H}$-module. If $f$ is an $\mathbf{H}$-valued continuously differentiable function on an open set in $E_{4}, \partial f$ is defined by

$$
\partial f(x)=1 \frac{\partial f}{\partial x^{0}}(x)-i \frac{\partial f}{\partial x^{1}}(x)-j \frac{\partial f}{\partial x^{2}}(x)-k \frac{\partial f}{\partial x^{3}}(x)
$$

$\partial f$ is a coordinate-free object, see Remark 2.9(ii). By analogy with the complex case we call $\partial$ the (left) Cauchy-Riemann operator. Similarly we introduce also $\bar{\partial}=\partial / \partial x^{0}+i \partial / \partial x^{1}+j \partial / \partial x^{2}+k \partial / \partial x^{3}$. Note that successive application of $\partial$ and $\bar{\partial}$ yields the Laplace operator, $\partial \bar{\partial}=\bar{\partial} \partial=\Delta$.

The inhomogeneous Cauchy-Riemann equation

$$
\partial A=F
$$

is our fundamental equation. To formulate the Euclidean covariance of this equation, we fix group actions. The 4-dimensional spin group is naturally realised in terms of quaternions. The set

$$
\operatorname{Sp}(1):=\{a \in \mathbf{H}:|a|=1\}
$$

carries a group structure inherited from the multiplicative group $\mathbf{H}^{\times}=\mathbf{H} \backslash\{0\}$ and is isomorphic to $S U(2)$. The group action

$$
(\operatorname{Sp}(1) \times \operatorname{Sp}(1)) \times \mathbf{H} \ni((a, b), x) \mapsto a x b^{-1} \in \mathbf{H}
$$

gives rise to an epimorphism $\mathrm{Sp}(1) \times \mathrm{Sp}(1) \rightarrow S O(4)$, the kernel of which is $\{ \pm(1,1)\}$ $\cong \mathbf{Z}_{2}$. Hence the isomorphism $(\operatorname{Sp}(1) \times \operatorname{Sp}(1)) / Z_{2} \cong S O(4)$. Furthermore the above action yields the semi-direct product of $\mathrm{Sp}(1) \times \mathrm{Sp}(1)$ and the additive group $\mathbf{H}$, denoted by $(\operatorname{Sp}(1) \times \operatorname{Sp}(1)) \odot H$.

If one wants to include reflections as well, one can proceed as follows. Let $\varrho$ be a group automorphism of $\mathrm{Sp}(1) \times \mathrm{Sp}(1)$ satisfying $\varrho \circ \varrho=i d$. $\varrho$ induces a semi-direct product $\mathbf{Z}_{2} \odot(\operatorname{Sp}(1) \times \operatorname{Sp}(1))$ with binary operation

$$
(-1,1,1)(1, a, b)=\left(-1, \varrho_{1}(a, b), \varrho_{2}(a, b)\right) .
$$


Since we regard $\mathbf{Z}_{2}$ as $O(4) / S O(4), \mathbf{Z}_{2} \odot(\operatorname{Sp}(1) \times \operatorname{Sp}(1))$ must act on $\mathbf{H}$ in the following manner:

$$
\begin{gathered}
(-1,1,1) \cdot x=\theta_{t}(x) \equiv-\bar{x}, \\
(1, a, b) \cdot x=a x b^{-1},
\end{gathered}
$$

where we choose the "time" reflection $\theta_{t}$ as a representative for $O(4) / S O(4)$. Comparing $(-1,1,1) \cdot\left(a x b^{-1}\right)$ and $((-1,1,1)(1, a, b)) x$, we have $\varrho(a, b)=(b, a)$ and hence

$$
(-1,1,1)(1, a, b)=(-1, b, a)=(1, b, a)(-1,1,1) .
$$

We have the following classification:

(2.4) Proposition. All possible four-dimensional (real) representations of $\mathrm{Sp}(1)$ $\times \mathrm{Sp}(1)$ are classified into the following 8 types (except for the trivial one)

$$
\begin{array}{llll}
(1 / 2,0) & a x, & \overline{(1 / 2,0)} & x a^{-1}=\overline{a \bar{x}} \\
(0,1 / 2) & b x, & \overline{(0,1 / 2)} & x b^{-1}=\overline{b \bar{x}} \\
(1 / 2,1 / 2) & a x b^{-1}, & \overline{(1 / 2,1 / 2)} & b x a^{-1}=\overline{a \bar{x} b^{-1}} \\
(0,0)+(1,0) & a x a^{-1}, & \\
(0,0)+(0,1) & b x b^{-1} .
\end{array}
$$

(2.5) Remark. In $(1 / 2,0),(0,1 / 2)$, and $(1 / 2,1 / 2)$, the latter ones are conjugate representations of the former ones. $(1 / 2,0)$ and $(0,1 / 2)$ are complex representations, since we can introduce a complex structure in $\mathbf{H}$ by right (respectively left) multiplication. Moreover, these are the so-called two-valued representations of $S O(4)$. On the other hand $(1 / 2,1 / 2),(0,0)+(1,0)$, and $(0,0)+(0,1)$ give rise to representations of $S O(4)$ itself. Finally we remark that $(1 / 2,1 / 2)$ (and its conjugate) is the only representation which admits extension to $\mathbf{Z}_{2} \odot(\operatorname{Sp}(1) \times \operatorname{Sp}(1))$ ). It is not possible to find $\theta \in G L(\mathbf{H})$ with $\theta((a, b) \cdot x)=(b, a) \cdot \theta(x)$ except for $(1 / 2,1 / 2)$ and $\overline{(1 / 2,1 / 2})$. This fact is also reflected in the failure of covariance of Eq. (2.2) under reflections.

A finite dimensional representation together with an action on a base manifold induces infinite dimensional representations on appropriate spaces of vectorvalued (generalised) functions. We shall use the same notations for the induced infinite dimensional representations of $(\operatorname{Sp}(1) \times \mathrm{Sp}(1)) \odot \mathrm{H}$. It is not difficult to show that $\partial$ and $\bar{\partial}$ intertwine the two representations $(1 / 2,0)$ and $(0,1 / 2)$.

The following commutative diagrams hold:

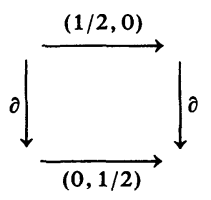

and

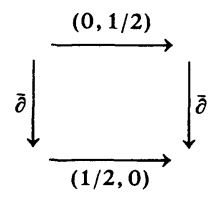


Similarly we have four other commutative diagrams

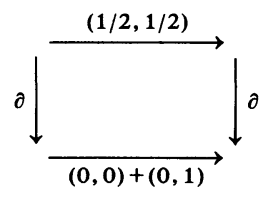

and

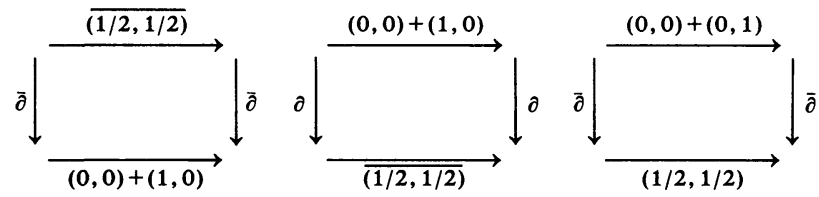

(2.9) Remarks. (i) If one wants to construct intertwining maps for $\overline{(1 / 2,0)}$ and $\overline{(0,1 / 2)}$, one has to consider the right instead of the left category. Incidentally, $(1 / 2,1 / 2), \overline{(1 / 2,1 / 2)},(0,0)+(1,0)$ and $(0,0)+(0,1)$ belong to the right category as well.

(ii) The operator $\partial$ is uniquely characterised up to real constants as a first order right $\mathbf{H}$-linear differential operator from the $\mathbf{H}$-module $C^{\infty}\left(E_{4} ; \mathbf{H}\right)$ into itself and intertwining $(1 / 2,0)$ and $(0,1 / 2)$ as in Eq. (2.6). Similarly for $\bar{\partial}$ and (2.7).

Our aim is to construct Euclidean field models by using random fields. Taking into account the known result of axiomatic field theory ([35]) on the relation between spin and statistics, we shall in the remainder of this paper be chiefly concerned with the pair $(1 / 2,1 / 2)$ and $(0,0)+(0,1)$. We shall assign the notation $A$ (respectively $F$ ) for a generic element of the (generalised) function space carrying the representation $(1 / 2,1 / 2)$ [respectively $(0,0)+(0,1)]$.

Our argument this far can be summarised as follows:

(2.10) Proposition. If $\partial A=F$, then $\partial(a \cdot A)=a \cdot F$ for any $\alpha \in(\operatorname{Sp}(1) \times \operatorname{Sp}(1)) \odot \mathbf{H}$.

We emphasise again that the above representations are those of $S O(4) \odot \mathbf{R}^{4}$, but we sacrifice the covariance under reflections.

(2.11) Remark. The direct sum $(1 / 2,0)+(0,1 / 2)$ admits extension to $\mathbf{Z}_{2} \odot(\operatorname{Sp}(1)$ $\times \mathrm{Sp}(1))$ and the pair $\{\partial, \partial\}$ forms the Dirac operator interchanging the grading of the $Z_{2}$-module $(1 / 2,0)+(0,1 / 2)$. Explicitly the Dirac equation can be written as a system of equations, namely $\partial A_{2}=F_{1}$ and $\bar{\partial} A_{1}=F_{2}$. In this representation the Dirac operator is the matrix $\not D=\left(\begin{array}{ll}0 & \partial \\ \delta & 0\end{array}\right)$.

Let us discuss the matter from a different angle. The exterior algebra $\Lambda_{*} \mathbf{R}^{4}$ is naturally isomorphic, as an $S O(4)$-module, to the direct sum $\mathbf{H} \oplus \mathbf{H} \oplus \mathbf{H} \oplus \mathbf{H}$ whose homogeneous components carry the representations $(1 / 2,1 / 2),(0,0)+(0,1),(0,0)$ $+(1,0)$, and $\overline{(1 / 2,1 / 2)}$, respectively. However, we must take a slight change of the grading of $\Lambda_{*} \mathbf{R}^{4}$. This will be described below.

Assuming that $\mathbf{R}^{4}$ is equipped with a Euclidean inner product $(\cdot, \cdot)_{E}$, we first choose a unit vector $e \in \mathbf{R}^{4}$. Then $\theta=\theta_{s} \in \operatorname{End}\left(\mathbf{R}^{4}\right)$ is uniquely determined by the properties

$$
\theta_{s}(e)=e, \quad \text { and } \quad \theta_{s}(x)=-x, \quad x \in\{e\}_{E}^{\perp}=\left\{x \in \mathbf{R}^{4}:(x, e)_{E}=0\right\} .
$$


We see that

$$
\theta_{s} \in O(4) \text { and } e \wedge \theta_{s}(x)=-e \wedge x, \quad x \in \mathbf{R}^{4} .
$$

In general any $\theta \in \operatorname{End}\left(\mathbf{R}^{4}\right)$ is naturally extended to $\operatorname{End}\left(\Lambda_{*} \mathbf{R}^{4}\right)$. In particular each element in $S O(4)$ gives rise to an automorphism of the graded algebra $\Lambda_{*} \mathbf{R}^{4}$.

Next, we fix an orientation of $\mathbf{R}^{4}$, i.e. we choose $0 \neq w \in \Lambda_{4} \mathbf{R}^{4}$. Combining this with the canonical inner product on $\Lambda_{*} \mathbf{R}^{4}$ lifted from $\mathbf{R}^{4}$, we may introduce the Hodge star operator as follows:

$$
\left.{ }_{E} u:=\|w\|^{-1} w\right\rfloor_{E} u, \quad u \in \Lambda_{*} \mathbf{R}^{4},
$$

where $\rfloor_{E}$ denotes interior multiplication defined by

$$
\left.\left(v_{1}\right\rfloor_{E} u, v_{2}\right)_{E}=\left(v_{1}, u \wedge v_{2}\right)_{E}, \quad v_{1}, v_{2} \in \Lambda_{*} \mathbf{R}^{4} .
$$

It is well known that $*_{E}$ maps $\Lambda_{2} \mathbf{R}^{4}$ into itself and that $\Lambda_{2} \mathbf{R}^{4}$ is decomposed into the eigenspaces of $*_{E}$ :

$$
\Lambda_{2} \mathbf{R}^{4}=\Lambda_{2,+} \mathbf{R}^{4} \oplus \Lambda_{2,-} \mathbf{R}^{4}=\{\text { self-dual 2-vectors }\} \oplus\{\text { anti-self dual 2-vectors }\} .
$$

At the same time this is the irreducible decomposition of the $S O(4)$-action and $\theta_{s}$ intertwines two irreducible components.

We now introduce another grading of $\Lambda_{*} \mathbf{R}^{4}$ as follows.

$$
\Lambda_{*} \mathbf{R}^{4}=\Lambda_{1} \mathbf{R}^{4} \oplus\left(\Lambda_{0} \mathbf{R}^{4} \oplus \Lambda_{2,-} \mathbf{R}^{4}\right) \oplus\left(\Lambda_{4} \mathbf{R}^{4} \oplus \Lambda_{2,+} \mathbf{R}^{4}\right) \oplus \Lambda_{3} \mathbf{R}^{4}
$$

The representations $(1 / 2,1 / 2),(0,0)+(0,1),(0,0)+(1,0)$, and $\overline{(1 / 2,1 / 2)}$ are realised on the homogeneous components. We shall explicitly construct the intertwining map between $(H,(0,0)+(0,1))$ and $\Lambda_{0} \mathbf{R}^{4} \oplus \Lambda_{2,-} \mathbf{R}^{4}$. To this end we introduce a binary operation $\circ$ on $\mathbf{R}^{4}$ as follows:

$$
x \circ y:=\left(\theta_{s}(x), y\right)_{E} e+\left(\left(\theta_{s}(x) \wedge y-*_{E}\left(\theta_{s}(x) \wedge y\right)\right)_{E}\right\rfloor_{E} e, \quad x, y \in \mathbf{R}^{4} \subset \Lambda_{*} \mathbf{R}^{4} .
$$

It is not difficult to verify that the linear space $\mathbf{R}^{4}$ with $\circ$ forms an algebra isomorphic to $\mathbf{H}$, where $e$ is the multiplicative identity and $\theta_{s}$ is the conjugation. If we define a map $\mathbf{R}^{4} \rightarrow \Lambda_{0} \mathbf{R}^{4} \oplus \Lambda_{2,-} \mathbf{R}^{4}$ by

$$
\Phi: x \mapsto x\rfloor_{E} e+e \wedge x-*_{E}(e \wedge x),
$$

then we see that $\Phi$ is a linear isomorphism satisfying

$$
\left.\Phi^{-1}(u)=e \wedge u_{0}+u_{2}\right\rfloor_{E} e,
$$

and

$$
\left\|\Phi^{-1}(u)\right\|_{E}^{2}=\left\|u_{0}\right\|_{E}^{2}+\frac{1}{2}\left\|u_{2}\right\|_{E}^{2}, \quad u=u_{0}+u_{2} \in \Lambda_{0} \mathbf{R}^{4} \oplus \Lambda_{2,-} \mathbf{R}^{4} .
$$

(2.12) Proposition. We have the identities

$$
\left.\Phi\left(\theta_{s}(x) \circ u\right)=u\right\rfloor_{E} x+x \wedge u-*_{E}(x \wedge u)
$$

and

$$
\left.x \circ \Phi^{-1}(v)=x \wedge v_{0}+v_{2}\right\rfloor_{E} x,
$$

for $x \in \mathbf{R}^{4}, u \in \Lambda_{1} \mathbf{R}^{4}$, and $v=v_{0}+v_{2} \in \Lambda_{0} \mathbf{R}^{4} \oplus \Lambda_{2,-} \mathbf{R}^{4}$. 
(2.13) Remark. That $\Phi$ intertwines $(\mathbf{H},(0,0)+(0,1)))$ and $\Lambda_{0} \mathbf{R}^{4} \oplus \Lambda_{2,-} \mathbf{R}^{4}$ is an immediate consequence of Proposition 2.11. It gives also an alternative description of the Cauchy-Riemann operator in terms of differential forms. Let us agree to write $\Gamma\left(\Lambda^{p} E_{4}\right)$ for the $C^{\infty}$ sections of the vector bundle $\Lambda^{p} E_{4}$ of all $p$-forms on $E_{4}$. Identifying $\Lambda^{p} E_{4}$ with $\Lambda_{p} E_{4}$ through the inner product $(\cdot, \cdot)_{E}$, we have

$$
\Phi(\partial \circ A)=-\delta_{E} A+d A-*_{E} d A, \quad A \in \Gamma\left(\Lambda^{1} E_{4}\right),
$$

and

$$
\bar{\delta} \circ \Phi^{-1}(F)=d F_{0}-\delta_{E} F_{2}, \quad F \in \Gamma\left(\Lambda^{0} E_{4} \oplus \Lambda^{2,-} E_{4}\right),
$$

where $\delta_{E}$ is the coderivative operator. Therefore our fundamental equation reads

$$
\delta_{E} A=-F_{0}, \quad d A-*_{E} d A=F_{2} .
$$

\section{Invariant Random Fields}

Let us now discuss the fundamental solution of $\bar{\partial}$ which is constructed from that of $-\Delta, g(x)=1 /\left(4 \pi^{2}|x|^{2}\right)$. We denote by $\mathscr{S} \equiv \mathscr{S}\left(E_{4}, \mathbf{H}\right)$ the Schwartz space of rapidly decreasing functions which we regard as carrying the representation $(1 / 2,1 / 2)$ unless otherwise stated. We introduce another space

$$
\mathscr{T}:=\left\{\varphi \in C^{\infty}\left(E_{4}, \mathbf{H}\right): \lim _{|x| \rightarrow \infty} \varphi(x)=0, \bar{\varphi} \varphi \in \mathscr{S}\right\} .
$$

We see that

$$
\mathscr{T} \ni \varphi \mapsto \bar{\partial} \varphi \in \mathscr{S}
$$

is bijective with inverse

$$
\mathscr{S} \ni \xi \mapsto-g * \partial \xi \in \mathscr{T}
$$

where

$$
g * \partial \xi(x)=\int_{E_{4}} g(x-y) \partial \xi(y) d y .
$$

The above bijection induces a locally convex topology and also the representation $(0,0)+(0,1)$ on $\mathscr{T} .(\operatorname{Sp}(1) \times \operatorname{Sp}(1)) \odot \mathbf{H}$ acts naturally on $\mathscr{T}^{\prime}$ and $\mathscr{S}^{\prime}$, the topological dual spaces of $\mathscr{T}$ and $\mathscr{S}$ respectively, through canonical pairings. It is important to note that $\{\bar{\partial} \varphi: \varphi \in \mathscr{S}\}$ is not dense in $\mathscr{S}$. Therefore the canonical injection $\imath: \mathscr{S} \hookrightarrow \mathscr{T}$ is not dense and furthermore $\imath^{*}: \mathscr{T}^{\prime} \rightarrow \mathscr{S}^{\prime}$ is not injective.

We shall now consider the equation $\partial A=F$ in the case where $F$ is a generalised random field. More precisely $F$ is a system of random variables $\{F(\varphi): \varphi \in \mathscr{T}\}$ defined on some probability space $(\Omega, \mathscr{F}, \mathbf{P})$ with the following properties:

$$
\mathbf{P}\left\{F\left(c_{1} \varphi_{1}+c_{2} \varphi_{2}\right)=c_{1} F\left(\varphi_{1}\right)+c_{2} F\left(\varphi_{2}\right)\right\}=1, \quad c_{1}, c_{2} \in \mathbf{R}, \varphi_{1}, \varphi_{2} \in \mathscr{T},
$$

and

$$
\mathscr{T}_{\ni} \varphi_{n} \rightarrow \varphi \in \mathscr{T} \Rightarrow F\left(\varphi_{n}\right) \rightarrow F(\varphi),
$$

where the latter convergence is in probability. 
We identify $F$ and $F^{\prime}$ if $F(\varphi)=F^{\prime}(\varphi)$ a.s. for each $\varphi \in \mathscr{T}$. Since $\mathscr{T}$ inherits the nuclear topology from $\mathscr{S}$, according to Minlos-Sazonov-Kolmogorov's theorem and the facts stated in the lemma below, one can always construct a unique $\mathscr{T}^{\prime}$-valued random variable $\widetilde{F}$ on the same probability space such that $\langle\varphi, \widetilde{F}\rangle$ $=F(\varphi)$ a.s. for every $\varphi \in \mathscr{T}$. (See Itô [26], for instance.)

Let us assume that we are given a countable family of random variables $\left\{X_{i}\right\}_{i=1}^{\infty}$ on $(\Omega, \mathscr{F}, \mathbf{P})$. The following result follows from known facts about Borel isomorphisms of measurable spaces; see e.g. Royden [33] or Yamasaki [39].

(3.1) Lemma. If there exists a countable generating system $\left\{f_{i}\right\}_{i=1}^{\infty}$ of measurable functions on a standard measurable space $(E, \mathscr{B})$, and a probability measure $\mu$ on $(E, \mathscr{B})$ such that the discrete time stochastic process $\left(E, \mathscr{B}, \mu,\left\{f_{i}\right\}\right)$ is equivalent to $\left(\Omega, \mathscr{F}, P,\left\{X_{i}\right\}\right)$, then it is possible to construct an E-valued random variable $X$ on $(\Omega, \mathscr{F}, P)$ such that $X_{i}=f_{i}(X)$ a.s. for each $i=1,2, \ldots$ In addition $X$ is unique up to null events.

(3.2) Remarks. (i) From now on we shall identify $F$ with $\widetilde{F}$ and write $\langle\varphi, F\rangle$ for $F(\varphi)$.

(ii) The index vector space $\mathscr{T}$ is somewhat peculiar in contrast with those usually taken. For instance, $\mathscr{T}$ does not admit a (smooth) partition of unity. This fact really makes the matter difficult in discussing the Markov property of $A$ solving $\partial A=F$ in general contexts (cf. Kusuoka [31]).

(3.3) Definition. A (generalised) random field $X$ is called Euclidean invariant if $a \cdot X$ is equivalent to $X$ in the sense of law for all $a \in(\operatorname{Sp}(1) \times \operatorname{Sp}(1)) \odot H$. We shall write $a \cdot X \stackrel{d}{=} X$ to indicate this. In the case $X=F$ one has to verify that $\left\langle a^{-1} \cdot \varphi, F\right\rangle \stackrel{d}{=}\langle\varphi, F\rangle$ for each $\varphi \in \mathscr{T}$.

(3.4) Remark. In the literature on random fields a stronger condition is often imposed, viz. the existence of a measure preserving flow

$$
\left\{T_{a} ; a \in(\mathrm{Sp}(1) \times \mathrm{Sp}(1)) \odot \mathbf{H}\right\}
$$

satisfying

$$
\mathbf{P}\left\{a \cdot F(\omega)=F\left(T_{a} \omega\right), \forall a\right\}=1 .
$$

In particular this is the case if $F$ is canonically realised.

The content of the following result from [12] is now clear.

(3.5) Theorem. Suppose that a generalised random field $F$ indexed by $\mathscr{T}$ is given on a probability space on $(\Omega, \mathscr{F}, \mathbf{P})$. Then there exists a unique generalised random field $\{\langle\xi, A\rangle: \xi \in \mathscr{S}\}$ defined on $(\Omega, \mathscr{F}, \mathbf{P})$ such that

$$
-\langle\bar{\partial} \varphi, A\rangle=\langle\varphi, F\rangle \text { a.s., } \varphi \in \mathscr{T} .
$$

Moreover if $F$ is Euclidean invariant, so is $A$.

The basic example of $F$ is white noise. Let $\psi$ be a continuous negative definite function (see Berg-Forst [16]) on $\mathbf{H}$ which is invariant under the action $(0,0)$ $+(0,1)$ of $\mathrm{Sp}(1) \times \mathrm{Sp}(1)$. The Lévy-Khinchin representation of $\psi$ must be of the 
following form:

$$
\begin{aligned}
\psi(\lambda)= & \sqrt{-1} \lambda_{0} \beta+\frac{\sigma_{0}}{2} \lambda_{0}^{2}+\frac{\sigma}{2}|\vec{\lambda}|^{2} \\
& +\int_{\mathbf{H} \backslash\{0\}}\left(1+\sqrt{-1}(\lambda, \alpha) 1_{(0,1)}(|\alpha|)-e^{\sqrt{-1}(\lambda, \alpha)}\right) v(d \alpha),
\end{aligned}
$$

where $\beta \in \mathbf{R}, \sigma_{0}, \sigma \geqq 0$, and the Lévy-measure $v$ is Ad-invariant under $\operatorname{Sp}(1)$, i.e. satisfies

$$
v\left(b d \alpha b^{-1}\right)=v(d \alpha), \quad b \in \operatorname{Sp}(1)
$$

(See also Albeverio et al. [8,9].) We shall only consider $\psi$ with this invariance.

Then

$$
C_{\psi}(\varphi):=\exp \left(-\int_{E_{4}} \psi(\varphi(x)) d x\right), \quad \varphi \in C_{0}^{\infty}\left(E_{4}, \mathbf{H}\right),
$$

is the characteristic functional of some Euclidean invariant generalised random field $\{\langle\varphi, N\rangle: \varphi \in \mathscr{D}\}$ indexed by the Schwartz space $\mathscr{D}$ of test functions carrying the representation $(0,0)+(0,1)$. Let us avoid detailed discussions about the continuity of $C_{\psi}$ with respect to the $\mathscr{T}$-topology, and just give a sufficient condition.

By Sobolev's inequality

$$
\|g * \partial \xi\|_{L^{p}} \leqq\left\{\int_{E_{4}}\left|\int_{E_{4}} \frac{|\xi(y)|}{2 \pi^{2}|x-y|^{3}} d y\right|^{p} d x\right\}^{1 / p} \leqq C_{p}\|\xi\|_{L^{q}}, \quad \xi \in \mathscr{S}
$$

if

$$
p>\frac{4}{3} \text { and } \frac{1}{q}=\frac{1}{p}+\frac{1}{4} .
$$

Therefore, if for some $\varepsilon>0$,

$$
\psi(\lambda)=O\left(|\lambda|^{4 / 3+\varepsilon}\right), \quad \lambda \rightarrow 0,
$$

$C_{\psi}(\varphi)=C_{\psi}(g * \partial(-\bar{\partial} \varphi))$ is well defined for all $\varphi \in \mathscr{T}$ and continuous in the $\mathscr{T}$-topology.

(3.8) Definition. We shall call a negative definite function $\psi$ satisfying (3.7) admissible.

Although in general there may exist two non-equivalent $\mathscr{T}^{\prime}$-valued random variables such that $\imath^{*} F=\imath^{*} F^{\prime}$ a.s., $N$ uniquely determines $F$ with $\imath^{*} F=N$, provided $\psi$ is admissible. In this sense we call $F$ the white noise with Lévy characteristic $\psi$.

We have the following result from [12] on our random field $A$.

(3.9) Theorem. Given an admissible Euclidean invariant continuous negative definite function $\psi$ on $(\mathbf{H},(0,0)+(0,1))$, there exists a generalised random field $\{\langle\xi, A\rangle: \xi \in \mathscr{S}\}$, unique up to law equivalence, such that $\{-\langle\delta \varphi, A\rangle: \varphi \in \mathscr{T}\}$ is the white noise with Lévy characteristic $\psi$. The distribution of $A$ is given by

$$
C_{A}(\xi):=\mathbf{E}\left[e^{\sqrt{-1}\langle\xi, A\rangle}\right]=\exp \left(-\int_{E_{4}} \psi(g * \partial \xi(x)) d x\right), \quad \xi \in \mathscr{S}
$$


and therefore $A$ is Euclidean invariant under the action $(1 / 2,1 / 2)$. If the Lévy measure has moments of order $n$ for $n=2,3, \ldots$, then also $A$ has moments of order $n$ :

$$
S_{n}\left(\xi_{1}, \ldots, \xi_{n}\right):=\mathbf{E}\left[\left\langle\xi_{1}, A\right\rangle \ldots\left\langle\xi_{n}, A\right\rangle\right], \quad \xi_{1}, \ldots, \xi_{n} \in \mathscr{S},
$$

exist as continuous linear functionals on $\mathscr{S}^{\otimes n}$.

The random field $A$ described in the theorem will be referred to as the solution of the quaternionic (inhomogeneous) Cauchy-Riemann equation $\partial A=F$ with Lévy characteristics $\psi$. We shall call $\left\{S_{n}\right\}$ the Schwinger functions of the random field $A$.

(3.10) Remark. In the pure Poisson case $\beta=\sigma_{0}=\sigma=0$, and with $v$ of finite variation, one can solve the equation $\partial A=F$ directly and a random field with analytic "paths" is obtained. We shall come back to representations of this kind in our forthcoming paper. (In this connection, see also [12].)

In the remainder of this section we shall discuss reflection invariance of $A$ (cf. [12]). We first consider sufficient conditions for reflection invariance and after that we shall state the complete result.

$A$ is by definition said to be (time-) reflection invariant if $\theta_{t} A \stackrel{d}{=} A$, with $\theta_{t}$ as in (2.3). It is easily seen that the following subdiagram of (2.8)

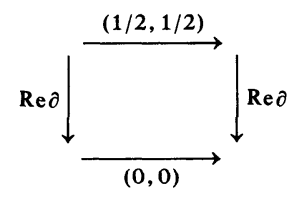

can be extended to $\mathbf{Z}_{2} \odot(\operatorname{Sp}(1) \times \operatorname{Sp}(1))$ consistently with $\theta_{t}$. This implies

$$
\operatorname{Re} g * \partial \theta_{t} \xi(x)=\operatorname{Re} g * \partial \xi\left(\theta_{t}^{-1} x\right), \quad \xi \in \mathscr{S}, x \in E_{4} .
$$

Therefore, if the Lévy characteristic $\psi$ depends only on $\operatorname{Re} \lambda$, i.e. $\sigma=0$ and $v$ is supported by $\left\{\alpha \in \mathbf{H}^{\times}: \operatorname{Im} \alpha=0\right\}$, then $\theta_{t} A \stackrel{d}{=} A$.

We next observe

$$
\int_{E_{4}}|g * \partial \xi(x)|^{2} d x=\iint_{E_{4} \times E_{4}} g(x-y)(\xi(x), \xi(y)) d x d y, \quad \xi \in \mathscr{S},
$$

for $\partial \bar{\partial}=\bar{\partial} \partial=\Delta$. Thus we see

$$
\int_{E_{4}}\left|g * \partial \theta_{t} \xi(x)\right|^{2} d x=\int_{E_{4}}|g * \partial \xi(x)|^{2} d x .
$$

Combining with (3.12) we deduce

$$
\int_{E_{4}}\left|\operatorname{Im} g * \partial \theta_{t} \xi(x)\right|^{2} d x=\int_{E_{4}}|\operatorname{Im} g * \partial \xi(x)|^{2} d x .
$$

The relations (3.12) and (3.14) tell us that the second order moment of the generalised random field $A$ is invariant under reflection. In particular, if $A$ is Gaussian distributed, i.e. $v=0$, then $\theta_{t} A \stackrel{d}{=} A$.

We can now formulate

(3.15) Theorem. The solution $A$ of the quaternionic Cauchy-Riemann equation $\partial A=F$ with admissible Lévy characteristic $\psi$ is time reflection invariant if and only if the Lévy measure $v$ is supported by the centre of $\mathbf{H}^{\times}$. 
Proof. We have already proved the "if" part. To prove the "only if" part, we start with the relation

$$
\operatorname{Re} \int_{E_{4}}\left(\psi\left(f_{1}(x)+f_{2}(x)\right)+\psi\left(f_{1}(x)-f_{2}(x)\right)\right) m(d x)=2 \operatorname{Re} \int_{E_{4}}\left(\psi\left(f_{1}(x)\right)+\psi\left(f_{2}(x)\right)\right) m(d x)
$$

which implies

$$
\operatorname{supp} v \subset\left\{\alpha:\left(\alpha, f_{1}(x)\right) \equiv 0 \bmod 2 \pi \text { or }\left(\alpha, f_{2}(x)\right) \equiv 0 \bmod 2 \pi\right\}, \quad m \text {-a.e. } x .
$$

Here $m \geqq 0$ is a general measure.

To see this implication, we may assume that

$$
\psi(\lambda)=\int_{\mathbf{H} \backslash\{0\}}(1-\cos (\alpha, \lambda)) v(d \alpha)
$$

It follows easily that then

$$
\begin{aligned}
& 2 \psi\left(\lambda_{1}\right)+2 \psi\left(\lambda_{2}\right)-\psi\left(\lambda_{1}+\lambda_{2}\right)-\psi\left(\lambda_{1}-\lambda_{2}\right) \\
& \quad=\int_{\mathbf{H} \backslash\{0\}}\left(1-\cos \left(\alpha, \lambda_{1}\right)\right)\left(1-\cos \left(\alpha, \lambda_{2}\right)\right) v(d \alpha) \geqq 0,
\end{aligned}
$$

with equality if and only if

$$
\left(\alpha, \lambda_{1}\right) \equiv 0 \bmod 2 \pi \quad \text { or } \quad\left(\alpha, \lambda_{2}\right) \equiv 0 \bmod 2 \pi, \quad \forall \alpha \in \operatorname{supp} v .
$$

The assertion now follows upon integration with respect to $m$.

By assumption $v$ is Ad-invariant under $\mathrm{Sp}(1)$, so (3.17) cannot hold unless one of the $\operatorname{Im} \lambda_{j} \operatorname{sis} 0$ or $v\left(\left\{\alpha \in \mathbf{H}^{\times}: \operatorname{Im} \alpha \neq 0\right\}\right)=0$. Hence if $(3.16)$ holds for $m(d x)=d x$ and the $f$ s are continuous, we deduce that

$$
\operatorname{supp} \operatorname{Im} f_{1} \cap \operatorname{supp} \operatorname{Im} f_{2} \text { has no interior, or } v\left(\left\{\alpha \in \mathbf{H}^{\times}: \operatorname{Im} \alpha \neq 0\right\}\right)=0 \text {. }
$$

After these preparations we turn to reflection invariance. If $A$ is reflection invariant, then one easily checks that $F$ must be invariant under the mapping

$$
\varphi \mapsto-g *(\bar{\partial} \bar{\varphi} \partial) \circ \theta_{t}^{-1} \text {. }
$$

We therefore define

$$
T \varphi=-g *(\bar{\partial} \bar{\varphi} \partial)
$$

Then

$$
\operatorname{Re} T \varphi(x)=\operatorname{Re} \varphi(x), \quad \operatorname{Im} T \varphi(x)=g *(\bar{\partial}(\operatorname{Im} \varphi) \partial)(x) .
$$

Note that if $\varphi \in C_{0}^{\infty}$, say, then $T \varphi$ is harmonic and therefore real-analytic off $\operatorname{supp} \varphi$. We shall show that there is a $\varphi_{1} \in C_{0}^{\infty}$ and

$$
\exists x \notin \operatorname{supp} \varphi_{1}: T \varphi_{1}(x) \neq 0 .
$$

That is, $T$ is non-local. (The condition in (3.19) is equivalent to requiring that $\operatorname{Im} T \varphi_{1}(x) \neq 0$.) Then by analyticity, $T \varphi_{1}$ cannot vanish on any open subset of the complement of $\operatorname{supp} \varphi_{1}$.

Assuming (3.19) for the moment, we define $\varphi_{2}=\varphi_{1}\left(\cdot+x_{0}\right)$, and let $S_{i}$ denote the support of $\varphi_{i}$. For $\left|x_{0}\right|$ sufficiently large, $S_{1} \cap S_{2}=\emptyset$, whereas $\operatorname{supp} \operatorname{Im} T \varphi_{1}$ nupp $\operatorname{Im} T \varphi_{2}$ must contain non-empty open subsets of the complement of $S_{1} \cup S_{2}$. 
If $A$ is reflection invariant, then $\int \psi(\varphi) d x=\int \psi(T \varphi) d x$ for any test function $\varphi$. Hence we see from (3.18) with $f_{i}=T \varphi_{i}$, that in order not to obtain a contradiction, $v$ must be supported by the real quaternions, i.e. by $Z\left(\mathbf{H}^{\times}\right)$, so the "only if" part follows.

Let us now prove (3.19). Suppose the contrary holds. Then $T$ is a local, continuous and linear operator $C_{0}^{\infty} \rightarrow C^{\infty}$. It is also translation invariant, hence a differential operator with constant coefficients. Only the zero order term can be non-zero, because $T$ is homogeneous of order zero. We have already seen that $T$ as an operator on $L^{2}$ is unitary. Consequently, $T$ is realised by multiplication with an element in $O(4)$. We know that $T$ preserves the real part. It follows that there is some $c \in \operatorname{Sp}(1)$ such that

$$
T \varphi=c \varphi c^{-1}, \quad \text { or } \quad T \varphi=c \bar{\varphi} c^{-1},
$$

for any test function $\varphi$.

Now we note that the diagram

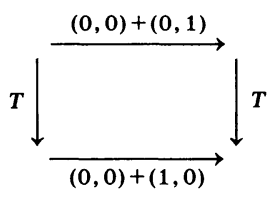

commutes. Supposing that $T$ satisfies the first identity in (3.20), we therefore get

$$
a c \varphi\left(a^{-1} x b\right) c^{-1} a^{-1}=c b \varphi\left(a^{-1} x b\right) b^{-1} c^{-1}, \quad x \in E_{4}
$$

for all $a, b \in \mathrm{Sp}(1)$, and $\varphi \in C_{0}^{\infty}\left(E_{4} ; \mathbf{H}\right)$. It is easily seen that this is not possible.

(3.21) Remarks. (i) Minor modifications of the argument just given show that in order for $T F$ to have independent values at each point when $F$ is a Poisson white noise, $T$ must be local.

(ii) In the above discussions we have seen that the behaviour of the solution $A$ of $\partial A=F$ with pure Poisson noise $F$ under reflection is compatible with the fact that the commutative diagram (2.8) does not permit any extension to $Z_{2} \odot(S p(1)$ $\times \mathrm{Sp}(1))$ while its subdiagram (3.11) does. On the other hand, provided that $F$ is Gaussian $A$ is always reflection invariant. In this case locality of $T$ itself does not really enter. What matters is the locality of $T T^{*}$ which equals the identity in this particular case, hence is local.

It seems true that the Gaussian case is exceptional also from some other observations concerning the Markov property, scaling property etc. We shall develop these topics in our forthcoming paper. (See also the comments at the end of Sect. 4.)

\section{Schwinger Functions and Wightman Functions}

In this section our attention is focused on the moments of the random field $A$ with characteristic $\psi$ from Theorem 3.9. We shall assume throughout that in addition to being admissible, $\psi$ is infinitely differentiable in some neighbourhood of $\lambda=0$.

Let $X_{1}, \ldots, X_{n}$ be a finite family of random variables on a probability space $(\Omega, \mathscr{F}, \mathbf{P})$. Assuming that their joint characteristic function is $n$ times continuously 
differentiable at the origin, we set

$$
U_{n}\left[X_{1}, \ldots, X_{n}\right]:=\left.(\sqrt{-1})^{-n} \frac{\partial^{n}}{\partial u_{1} \ldots \partial u_{n}} \log \mathbf{E}\left[e^{\sqrt{-1} u_{1} X_{1}+\ldots+\sqrt{-1} u_{n} X_{n}}\right]\right|_{u_{1}=\ldots=u_{n}=0},
$$

and call it the cumulant of $X_{1}, \ldots, X_{n}$.

(4.1) Lemma. $U_{n}$ is a symmetric n-linear form on the linear space $L^{n}(\Omega, \mathscr{F}, \mathbf{P})$ of all random variables on $(\Omega, \mathscr{F}, \mathbf{P})$ with finite $n^{\text {th }}$ moments. Moments and cumulants are related as follows. Let $\mathscr{P}$ denote all partitions $\Delta=\{I\}$ of $\{1, \ldots, n\}$. Then, writing $I=\left\{i_{1}, \ldots, i_{m}\right\}$,

$$
\mathbf{E}\left[X_{1} \ldots X_{n}\right]=\sum_{\Delta \in \mathscr{P}} \prod_{I \in \Delta} U_{m}\left[X_{i_{1}}, \ldots, X_{i_{m}}\right] \text {. }
$$

Proof. It suffices to consider the case $X_{1}=\ldots=X_{n}=X$, due to the obvious symmetry and multilinearity. The group of permutations of $\{1, \ldots, n\}$ acts naturally on $\mathscr{P}$. Since the order of the isotropy group of $\Delta \in \mathscr{P}$ equals $\prod_{m=1}^{n} i_{m} !(m !)^{i_{m}}$, where $i_{m}$ is the number of $I$ 's in $\Delta$ with $m$ elements, $1 \leqq m \leqq n$, one obtains

$$
\begin{aligned}
M_{n} & \equiv \text { right-hand side of Lemma (4.1) } \\
& =\sum_{\substack{i_{1}, \ldots, i_{m} \\
i_{1}+2 i_{2}+\ldots+n i_{n}=n}} \frac{n !}{\prod_{m=1}^{n} i_{m} !(m !)^{i_{m}}} \prod_{m=1}^{n} U_{m}[X, \ldots, X]^{i_{m}},
\end{aligned}
$$

and hence

$$
\begin{aligned}
& \exp \left\{\sum_{m=1}^{n} \frac{(\sqrt{-1})^{m}}{m !} U_{m}[X, \ldots, X] z^{m}\right\} \\
& =1+\sum_{m=1}^{n} \frac{(\sqrt{-1})^{m}}{m !} M_{m} z^{m}+o\left(|z|^{n}\right), \quad z \rightarrow 0 .
\end{aligned}
$$

This completes the proof.

Under our assumptions on $\psi$, the Lévy measure $v$ has moments of all orders $n \geqq 2$. From the formulae

$$
\int_{\mathbf{H} \backslash\{0\}} \alpha_{0}^{n-m}(\vec{\lambda} \cdot \vec{\alpha})^{m} v(d \alpha)=\frac{1}{m+1} \int_{\mathbf{H} \backslash\{0\}} \alpha_{0}^{n-m}|\vec{\alpha}|^{m} v(d \alpha)|\vec{\lambda}|^{m}, \quad m=0,2, \ldots,
$$

and

$$
\int_{\mathbf{H} \backslash\{0\}} \alpha_{0}^{n-m}(\vec{\lambda} \cdot \vec{\alpha})^{m} v(d \alpha)=0, \quad m=1,3, \ldots,
$$

which are consequences of the $(0,0)+(0,1)$-symmetry of $v$, we obtain the following Taylor expansion of $\psi$ :

$$
\begin{aligned}
-\psi(\lambda)= & -\frac{1}{2}\left(\sigma_{0}+\int_{\mathbf{H} \backslash\{0\}} \alpha_{0}^{2} v(d \alpha)\right) \lambda_{0}^{2}-\frac{1}{2}\left(\sigma+\frac{1}{3} \int_{\mathbf{H}\{\{0\}}|\vec{\alpha}|^{2} v(d \alpha)\right)|\vec{\lambda}|^{2} \\
& +\sum_{n=3}^{N} \frac{(\sqrt{-1})^{n}}{n !} \sum_{\substack{m=0 \\
m=0}}^{n}\left(\begin{array}{c}
n \\
m
\end{array}\right) \frac{1}{m+1} \\
& \times \int_{\mathbf{H} \backslash\{0\}} \alpha_{0}^{n-m}|\vec{\alpha}|^{m} v(d \alpha) \lambda_{0}^{n-m}|\vec{\lambda}|^{m}+o\left(|\lambda|^{N}\right) .
\end{aligned}
$$


We remark here that in order for $\psi$ to be admissible, the first order term in its Taylor development must vanish.

Let $\xi \in \mathscr{S}$. Then

$$
\begin{aligned}
U_{2}(\langle\xi, A\rangle\langle\xi, A\rangle)= & \left(\sigma_{0}+\int_{\mathbf{H}\{\{0\}} \alpha_{0}^{2} v(d \alpha)\right) \int_{E_{4}}(\operatorname{Re} g * \partial \xi(x))^{2} d x \\
& +\left(\sigma+\frac{1}{3} \int_{\mathbf{H} \backslash\{0\}}|\vec{\alpha}|^{2} v(d \alpha)\right) \int_{E_{4}}|\operatorname{Im} g * \partial \xi(x)|^{2} d x,
\end{aligned}
$$

and

$$
\begin{aligned}
U_{n}(\langle\xi, A\rangle \ldots\langle\xi, A\rangle)= & \sum_{\substack{m=0 \\
m \text { even }}}^{n}\left(\begin{array}{c}
n \\
m
\end{array}\right) \frac{1}{m+1} \int_{\mathbf{H} \backslash\{0\}} \alpha_{0}^{n-m}|\vec{\alpha}|^{m} v(d \alpha) \\
& \times \int_{E_{4}}(\operatorname{Re} g * \partial \xi(x))^{n-m}|\operatorname{Im} g * \partial \xi(x)|^{m} d x, \quad n \geqq 3 .
\end{aligned}
$$

In what follows we shall discuss the problem of analytically continuing the moments of $A$ by letting $\left(x^{0}, x^{1}, x^{2}, x^{3}\right) \in E_{4}$ become complex.

Let us recall some fundamental notions and notations associated with Minkowski space. By definition Minkowski space $M_{4}$ is an affine space with difference space $\mathbf{R}^{4}$ equipped with an inner product $(\cdot, \cdot)_{M}$ of signature $(3,1)$. We denote by $Q$ the associated quadratic form on $\mathbf{R}^{4}$.

In the following arguments we intend to distinguish the space of linear forms $\Lambda^{1} \mathbf{R}^{4}=\operatorname{Hom}\left(\mathbf{R}^{4}, \mathbf{R}\right)$ from $\mathbf{R}^{4}$. One consequence of this is that interior multiplication is defined between elements of $\Lambda_{*} \mathbf{R}^{4}$ and elements of $\Lambda^{*} \mathbf{R}^{4}$, the alternating algebra of $\mathbf{R}^{4}$. There exists a unique map $\eta_{M} \in \operatorname{Hom}\left(\mathbf{R}^{4}, \Lambda^{1} \mathbf{R}^{4}\right)$ corresponding to the inner product $(\cdot, \cdot)_{M}$ through the relation

$$
\left\langle\eta_{M}(x), y\right\rangle=(x, y)_{M}, \quad x, y \in \mathbf{R}^{4} .
$$

Since $\eta_{M}$ is an isomorphism, the inner product $(\cdot, \cdot)_{M}$ is transferred to $\Lambda^{1} \mathbf{R}^{4}$ by $\eta_{M}$. We shall use the same notation for the induced inner product.

(4.3) Definition. A vector $x \in \mathbf{R}^{4}$ is said to be space-like, light-like or time-like according as $Q(x)$ is positive, zero, or negative.

The totality $V$ of time-like vectors has an easily deduced remarkable property, (ii) below. We shall need property (i) later on.

(4.4) Proposition. For $x, y, z \in V$,

(i) $(x, y)_{M}^{2} \geqq Q(x) Q(y)>0$, and

(ii) $(x, y)_{M}<0$ and $(y, z)_{M}<0$ imply $(x, z)_{M}<0$.

On account of (ii) the set $V$ is divided into two equivalence classes: $x, y \in V$ are called equivalent if $(x, y)_{M}<0$.

(4.5) Definition. We say that $\left(\mathbf{R}^{4},(\cdot, \cdot)_{M}\right)$ is oriented in time if a preferred choice of one of these classes is made. The chosen one is called the (open) forward cone. It will be denoted by $V^{+}$, whereas the remaining one will be denoted by $V^{-}$.

In the following discussion we fix a time orientation. Let $e_{0} \in V$. Then $Q$ is positive definite on the subspace $\left\{e_{0}\right\}_{M}^{\perp} \equiv\left\{x \in \mathbf{R}^{4}:\left(x, e_{0}\right)_{M}=0\right\}$. We regard $\mathbf{R}^{4}=\mathbf{R} e_{0} \oplus\left\{e_{0}\right\}_{M}^{\perp}$ as a decomposition of (the tangent space of ) Minkowski space into "time" and "space." Hereafter, by an orthogonal frame we shall mean a set of 
vectors $\left\{e_{0}, e_{1}, e_{2}, e_{3}\right\} \subset \mathbf{R}^{4}$ such that $Q\left(e_{0}\right)=-1$ and $\left\{e_{1}, e_{2}, e_{3}\right\}$ is an orthonormal basis of $\left\{e_{0}\right\}_{M}^{1}$.

We now consider complexification, $M_{4}^{\mathrm{C}}$, of the Minkowski space $M_{4}$.

More generally, suppose we are given an imbedding of a $C^{\infty}$ manifold $M$ in a complex manifold $M^{\mathbf{C}}$ with complex structure $J$ in such a way that the imbedding $\imath: M \hookrightarrow M^{\mathbf{C}}$ decompose the tangent space into real and imaginary parts:

$$
T_{\imath(p)} M^{\mathrm{C}}=l_{* p} T_{p} M \oplus J_{\imath(p)} l_{* p} T_{p} M, \quad p \in M,
$$

then we refer to $M^{\mathbf{C}}$ as a complexification of $M$.

By assigning $\mathscr{L}^{n} T_{p} M$, the vector space of complex-valued $n$-linear forms on $T_{p} M$, to each $p \in M$, we get a complex vector bundle

$$
\mathscr{L}^{n} T M=\bigcup_{p \in M} \mathscr{L}^{n} T_{p} M
$$

On $M^{\mathrm{C}}$ we consider the subbundle

$$
\mathscr{H}^{n} T M^{\mathrm{c}}=\bigcup_{p \in M} \mathscr{H}^{n} T_{p} M^{\mathrm{c}},
$$

consisting of all holomorphic elements of $\mathscr{L}^{n} T_{p} M^{\mathrm{c}}$, i.e.,

$$
\begin{aligned}
\mathscr{H}^{n} T_{p} M^{\mathbf{c}} & \equiv\left\{f \in \mathscr{L}^{n} T_{p} M^{\mathbf{c}}: f\left(u_{1}, \ldots, u_{i-1}, J_{p} u_{i}, u_{i+1}, \ldots, u_{n}\right)\right. \\
& \left.=\sqrt{-1} f\left(u_{1}, \ldots, u_{i}, \ldots, u_{n}\right), \forall u_{1}, \ldots, u_{n} \in T_{p} M^{\mathbf{c}}, \forall i=1, \ldots, n\right\} .
\end{aligned}
$$

(Cf. e.g. Kobayashi-Nomizu [30], vol. II.) This condition is equivalent to requiring that the multi C-linear extension of $f$ vanishes if at least one of the $u$ 's is of antiholomorphic type, i.e. $J u_{i}=-\sqrt{-1} u_{i}$ for some $1 \leqq i \leqq n$.

It follows from the decomposition (4.6) that for each $p \in M$ the mapping

$$
\tau\left(l_{*_{p}}\right): \mathscr{H}^{n} T_{\imath(p)} M^{\mathrm{C}} \ni f \mapsto f \circ l_{k_{p}} \in \mathscr{L}^{n} T_{p} M,
$$

where $f \circ l_{*_{p}}\left(u_{1}, \ldots, u_{n}\right)=f\left(l_{*} u_{1}, \ldots, l_{*} u_{n}\right)$ is a $\mathbf{C}$-linear isomorphism. Hence for every $f \in \mathscr{L}^{n} T_{p} M$ there exists a unique element $\widetilde{f} \in \mathscr{H}^{n} T_{\imath(p)} M^{\mathbf{C}}$ which makes the following diagram commute:

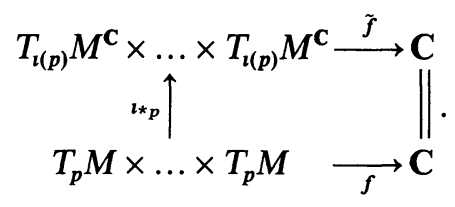

By the same reasoning we can associate each $\alpha \in \operatorname{Hom}_{\mathbf{c}}\left(\mathscr{L}^{n} T_{p} M, \mathscr{L}^{m} T_{p} M\right)$ with $\tilde{\alpha} \in \operatorname{Hom}_{\mathbf{C}}\left(\mathscr{H}^{n} T_{\imath(p)} M^{\mathbf{c}}, \mathscr{H}^{m} T_{\imath(p)} M^{\mathbf{c}}\right)$. $\tilde{\alpha}$ is uniquely characterised by the following commutative diagram:

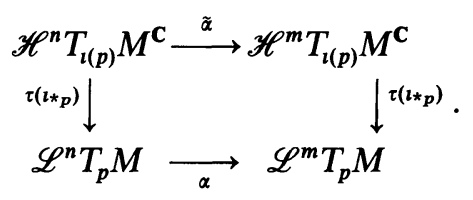

A local $C^{\infty}$ section $\widetilde{f}$ of $\mathscr{H}^{n} T M^{\mathbf{c}}$ is called holomorphic if the function $\widetilde{f}\left(u_{1}, \ldots, u_{n}\right)$ is holomorphic for any $n$ holomorphic vector fields $u_{1}, \ldots, u_{n}$. 
Suppose $f \in \Gamma\left(\mathscr{L}^{n} T M\right)$ is given. We say that a holomorphic section $\tilde{f}$ of $\mathscr{H}^{n} T M^{\mathbf{c}}$ defined on some neighbourhood of $\imath(M)$ is a holomorphic extension of $f$ if $\imath^{*} f=f$. According to the discussion above and the unique determination of a holomorphic function by its values on a real environment, $\tilde{f}$ is uniquely determined by its trace $\imath^{*} \widetilde{f}$ on $M$. (See e.g. Streater-Wightman [35] or Jost [29].) Similarly we say that a linear differential operator $\tilde{D}: \Gamma\left(\mathscr{H}^{n} T M^{\mathbf{C}}\right) \rightarrow \Gamma\left(\mathscr{H}^{m} T M^{\mathbf{C}}\right)$ is a holomorphic extension of a linear differential operator $D: \Gamma\left(\mathscr{L}^{n} T M\right)$ $\rightarrow \Gamma\left(\mathscr{L}^{m} T M\right)$ if $\tilde{D} \tilde{f}$ is holomorphic and $\imath^{*}(\tilde{D} f)=D\left(\imath^{*} \tilde{f}\right)$ for every holomorphic section $\tilde{f}$ of $\mathscr{H}^{n} T M^{\mathrm{c}}$.

Let $M_{4}^{\mathbf{C}}$ be a four-dimensional complex affine space with difference space $\mathbf{R}^{4} \otimes \mathbf{C}$, and denote by $J$ its natural complex structure. We assume that the Minkowski space $M_{4}$ is imbedded into $M_{4}^{\mathrm{C}}$ such that the embedding $l_{M}: M_{4} \hookrightarrow M_{4}^{\mathrm{C}}$ induces the natural imbedding of the difference space $\mathbf{R}^{4} \hookrightarrow \mathbf{R}^{4} \otimes \mathbf{C}$. Then $M_{4}^{\mathrm{C}}$ is a complexification of $M_{4}$ in the sense described above.

We consider now the holomorphic extension $\widetilde{Q}$ of the quadratic form $Q . \widetilde{Q}$ is determined as the quadratic form $\mathbf{R}^{4} \otimes \mathbf{C} \rightarrow \mathbf{C}$ satisfying the additional property

$$
\widetilde{Q}(z+J z)-\widetilde{Q}(z-J z)=4 \sqrt{-1} \tilde{Q}(z), \quad z \in \mathbf{R}^{4} \otimes \mathbf{C} .
$$

It follows that

$$
\widetilde{Q}(x+J y)=Q(x)-Q(y)+\frac{\sqrt{-1}}{2}(Q(x+y)-Q(x-y)), \quad x, y \in \mathbf{R}^{4} .
$$

For simplicity we shall drop the tilde and simply write $Q$ from now on. It is worthwhile noting that $Q(z) \in \mathbf{C} \backslash(-\infty, 0]$ if $z \in \mathbf{R}^{4}+J V^{+}$. This is a consequence of (i) in Proposition 4.4.

Let us recall that $\mathbf{H}$ is also regarded as the difference space of the Euclidean space $E_{4}$. We fix an imbedding $l_{E}: E_{4} \rightarrow M_{4}^{\mathrm{C}}$ which satisfies the following conditions:

$$
\left(l_{E}\right)_{*}(\mathbf{H})=\left\{e_{0}\right\}_{M}^{\perp} \oplus \mathbf{R} J e_{0}, \quad Q\left(\left(l_{E}\right)_{*} x\right)=|x|^{2}, \quad x \in \mathbf{H} .
$$

We next define several domains on which analytic continuation will be considered. Let $T^{n}$ be the tubular domain in $\left(M_{4}^{\mathbf{C}}\right)^{n}$ with base $V^{+}$, i.e.

$$
T^{n} \equiv\left\{\mathbf{z}=\left(z_{1}, \ldots, z_{n}\right) \in\left(M_{4}^{\mathrm{C}}\right)^{n}: z_{i+1}-z_{i} \in \mathbf{R}^{4}+J V^{+}, 1 \leqq i \leqq n-1\right\} .
$$

$T^{n}$ is customarily called the forward tube.

In addition we define two open sets in $\left(E_{4}\right)^{n}$ as follows:

$$
\left(E_{4}\right)_{\neq}^{n} \equiv\left\{\mathbf{x}=\left(x_{1}, \ldots, x_{n}\right) \in\left(E_{4}\right)^{n}: x_{i} \neq x_{j} \text { if } i \neq j\right\},
$$

and

$$
\left(E_{4}\right)_{<}^{n} \equiv\left\{\mathbf{x}=\left(x_{1}, \ldots, x_{n}\right) \in\left(E_{4}\right)^{n}: l_{E}(\mathbf{x})=\left(l_{E}\left(x_{1}\right), \ldots, l_{E}\left(x_{n}\right)\right) \in T^{n}\right\} .
$$

Analytic continuation of the Schwinger functions (moments) $\left\{S_{n}\left(\xi_{1}, \ldots, \xi_{n}\right)\right\}$ of the random field $A$ from Theorem 3.9 will be performed in accordance with the following diagram: 


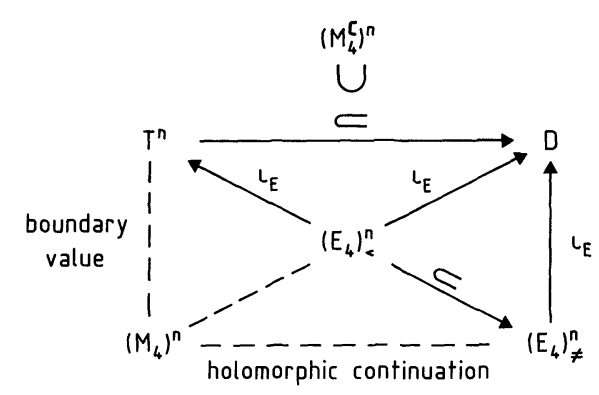

Since we have rather explicit formulae for the $S_{n}$ it is possible to exhibit the analyticity directly. We first show that the distribution $S_{n}$ on $\left(E_{4}\right)^{n}$ is representable as a pullback of a holomorphic section $\mathscr{W}_{n}$ of $\mathscr{H}^{n} T\left(M_{4}^{C}\right)^{n}$ onto $\left(E_{4}\right)_{\neq}^{n}$. We see next that $\mathscr{W}_{n}$ is analytically continued to the domain $D$ which contains the forward tube. Finally, by taking the boundary value of $\mathscr{W}_{n}$ we shall obtain (the Wightman function or distribution) $W_{n}$ on $M_{4}$.

(4.8) Remark. Besides our purpose of exploiting explicit formulae for the $W_{n}$, we are more or less forced to perform the analytic continuation by hand. It is not clear at the moment whether the standard procedures for analytic continuation from axiomatic quantum field theory due to Nelson, Osterwalder-Schrader and others, see e.g. Glimm-Jaffe [20], do apply in our model.

Let us now turn to an alternative description of the cumulants of $A$. Identification of $\mathbf{H}$ with the difference space of $E_{4}$ gives rise to the isomorphism $\mathscr{S}=\mathscr{S}\left(E_{4}, \mathbf{H}\right) \cong \mathscr{S}\left(T E_{4}\right)$. In terms of the notation introduced at the end of Sect. 2 ,

$$
\Phi(\partial u)=-\delta_{E} \eta_{E}(u)+d \eta_{E}(u)-*_{E} d \eta_{E}(u), \quad u \in \Gamma\left(T E_{4}\right),
$$

where $\eta_{E} \in \operatorname{Hom}\left(\mathbf{R}^{4}, \Lambda^{1} \mathbf{R}^{4}\right)$ corresponds to $(\cdot, \cdot)_{E}$ in the same way as in (4.2). Therefore, if $u \in \mathscr{S}\left(T E_{4}\right)$, we have

$$
\operatorname{Re} g * \partial u(x)=-g * \delta_{E} \eta_{E}(u)(x)=-\int_{E_{4}}\langle d g(x-\cdot)(y), u(y)\rangle d y
$$

and choosing an orthogonal basis $\left\{e^{0}, e^{1}, e^{2}, e^{3}\right\}$ of $\Lambda^{1} \mathbf{R}^{4}$ with respect to $(\cdot, \cdot)_{E}$, we get

$$
\begin{aligned}
|\operatorname{Im} g * \partial u(x)|^{2} & =\frac{1}{2}\left\|g *\left(d \eta_{E}(u)-*_{E} d \eta_{E}(u)\right)(x)\right\|_{E}^{2} \\
& =\sum_{i=1}^{3}\left|\int_{E_{4}} g(x-y)\left(e^{0} \wedge e^{i}-*_{E}\left(e^{0} \wedge e^{i}\right), d \eta_{E}(u)(y)\right)_{E} d y\right|^{2} \\
& =\sum_{i=1}^{3}\left|\int_{E_{4}}\left\langle\delta_{E}\left(\left(e^{0} \wedge e^{i}-*_{E}\left(e^{0} \wedge e^{i}\right)\right) \wedge g(x-\cdot)\right)(y), u(y)\right\rangle d y\right|^{2} \\
& =\int_{\left(E_{4}\right)^{2}}\left\langle D_{E}(g(x-\cdot) \otimes g(x-\cdot))\left(y_{1}, y_{2}\right), u \otimes u\left(y_{1}, y_{2}\right)\right\rangle d y_{1} d y_{2},
\end{aligned}
$$

where $D_{E}: C^{\infty}\left(E_{4}\right) \otimes C^{\infty}\left(E_{4}\right) \rightarrow \Gamma\left(\Lambda^{1} E_{4}\right) \otimes \Gamma\left(\Lambda^{1} E_{4}\right)$ is the differential operator defined by

$$
\begin{aligned}
D_{E}\left(f_{1} \otimes f_{2}\right)= & \sum_{i=1}^{3} \delta_{E}\left(\left(e^{0} \wedge e^{i}-*_{E}\left(e^{0} \wedge e^{i}\right)\right) \wedge f_{1}\right) \\
& \otimes \delta_{E}\left(\left(e^{0} \wedge e^{i}-*_{E}\left(e^{0} \wedge e^{i}\right)\right) \wedge f_{2}\right)
\end{aligned}
$$


Define also

$$
g^{(n)}(\mathbf{y})=g^{(n)}\left(y_{1}, \ldots, y_{n}\right)=\int_{E_{4}} g\left(x-y_{1}\right) \ldots g\left(x-y_{n}\right) d x, \quad \mathbf{y} \in\left(E_{4}\right)_{\mp}^{n} .
$$

(4.13) Remark. For $n \geqq 3$ the decrease at infinity of $g(y)=c|y|^{-2}$ is sufficient for integrability, i.e. $g^{(n)}$ is well defined on $\left(E_{4}\right)_{\neq}^{n}$ for $n \geqq 3$. For $n=2$ we must interpret (4.12) as the finite part of a divergent integral; cf. e.g. Hörmander [25], Chap. 3. Hence we understand that for $n=2$

$$
g^{(2)}\left(y_{1}, y_{2}\right)=-\frac{1}{8 \pi^{2}} \log \left|y_{1}-y_{2}\right| \text {. }
$$

It is clear from the behaviour of $g^{(n)}$ that the cases $n=2$ and $n \geqq 3$ are different. Accordingly we shall treat them separately, starting with the latter case $n \geqq 3$.

Using (4.9), (4.10) and Fubini's theorem we obtain

$$
\begin{aligned}
\int_{E_{4}} & (\operatorname{Re} g * \partial u(x))^{n-m}|\operatorname{Im} g * \partial u(x)|^{m} d x \\
= & \left.\int_{\substack{\left(E_{4}\right)^{n} \\
0 \leqq m}}\langle\operatorname{Sym} \overbrace{(d \otimes \ldots \otimes d}^{n-m} \otimes \overbrace{D_{E} \otimes \ldots \otimes D_{E}}^{n-m}) g^{(n)}(\mathbf{y}), u \otimes \ldots \otimes u(\mathbf{y})\right\rangle d \mathbf{y}, \\
& =\leqq m,
\end{aligned}
$$

where "Sym" denotes a symmetrisation which we shall now explain. The group of permutations of $n$ letters acts to the right on $\left(E_{4}\right)^{n}$ by

$$
R_{\sigma}(\mathbf{x})=\mathbf{x} \cdot \sigma=\left(x_{\sigma(1)}, \ldots, x_{\sigma(n)}\right), \quad \mathbf{x} \in\left(E_{4}\right)^{n} .
$$

$\operatorname{Sym}\left(d \otimes \ldots \otimes d \otimes D_{E} \otimes \ldots \otimes D_{E}\right)$ is the linear partial differential operator $C^{\infty}\left(\left(E_{4}\right)^{n}\right)$ $\rightarrow \Gamma\left(\mathscr{L}^{n} T\left(E_{4}\right)^{n}\right)$ defined by

$$
f \mapsto \frac{1}{n !} \sum_{\sigma}(R_{\sigma^{-1}}^{*} \overbrace{d \otimes \ldots \otimes d}^{n-m} \otimes \overbrace{D_{E} \otimes \ldots \otimes D_{E}}^{m / 2}\left(R_{\sigma}^{*} f\right)) .
$$

We first assume the existence of a holomorphic extension $G_{n}$ of $g^{(n)}=l_{E}^{*} G_{n}$ with domain containing the permuted extended forward tube - to be denoted by $T_{\text {p.e. }}^{n}$. hereafter - obtained by applying all complex Lorentz transformations and permutations to the forward tube $T^{n}$ in (4.7), and obtain a final formula for $W_{n}$, postponing for a while the discussion of existence itself.

Let $\omega_{E}$ be the constant section of $\Lambda^{4} E_{4}$ corresponding to the orientation of the difference space $\mathbf{H} . M_{4}$ is not oriented so far. Here we choose one of the normalised constant sections of $\Lambda^{4} E_{4}$, denoted by $\omega_{M}$. On $M_{4}$, the Hodge star operator is defined by

$$
\left.{ }_{M} f=-\omega_{M}\right\rfloor \eta_{M}^{-1}(f), \quad f \in \Lambda^{*} M_{4} .
$$

It is easily seen that the holomorphic extension $\tilde{\omega}_{M}$ satisfies either $l_{E}^{*} \tilde{\omega}_{M}=\sqrt{-1} \omega_{E}$ or $l_{E}^{*} \tilde{\omega}_{M}=-\sqrt{-1} \omega_{E}$. Correspondingly $\left(\tilde{*}_{M} f\right) \circ\left(l_{E}\right)_{*}= \pm \sqrt{-1} *{ }_{E}\left(f \circ\left(l_{E}\right)_{*}\right)$ for $f \in \Lambda^{n, 0} M_{4}^{\mathbf{C}}=\mathscr{H}^{n} T M_{4}^{\mathbf{C}} \cap \Lambda^{n} M_{4}^{\mathbf{C}}$. In any case the coderivative operator $\delta_{M}$ on $M_{4}$ defined by

$$
\delta_{M} f=(-1)^{4 n+4+1}(-1) *_{M} d *_{M} f=*_{M} d *_{M} f, \quad f \in \Gamma\left(\Lambda^{n} M_{4}\right),
$$

coincides with $\delta_{E}$ in the sense that its holomorphic extension $\widetilde{\delta}_{M}$ satisfies

$$
\imath_{E}^{*}\left(\tilde{\delta}_{M} f\right)=\delta_{E}\left(\imath_{E}^{*} f\right)
$$


for every holomorphic differential form $f$ on $M_{4}^{\mathrm{C}}$. Finally, combining the formulae above, we see that $D_{E}$ is analytically continued to the differential operator

$$
D_{M}: C^{\infty}\left(M_{4}\right) \otimes C^{\infty}\left(M_{4}\right) \rightarrow \Gamma\left(\Lambda^{1} M_{4}\right) \otimes \Gamma\left(\Lambda^{1} M_{4}\right)
$$

defined by

$$
\begin{aligned}
D_{M}\left(f_{1} \otimes f_{2}\right)= & -\sum_{i=1}^{3} \delta_{M}\left(\left(e^{0} \wedge e^{i}-\sqrt{-1} *_{M}\left(e^{0} \wedge e^{i}\right)\right) \wedge f_{1}\right) \\
& \otimes \delta_{M}\left(\left(e^{0} \wedge e^{i}-\sqrt{-1} *_{M}\left(e^{0} \wedge e^{i}\right)\right) \wedge f_{2}\right),
\end{aligned}
$$

if $\imath_{E}^{*} \tilde{\omega}_{M}=\sqrt{-1} \omega_{E}$. In the case $\imath_{E}^{*} \tilde{\omega}_{M}=-\sqrt{-1} \omega_{E}$, the minus signs in front of $\sqrt{-1} *_{M}$ change to plus signs. Here $\left\{e^{0}, e^{1}, e^{2}, e^{3}\right\}$ is the dual of an orthogonal frame $\left\{e_{0}, e_{1}, e_{2}, e_{3}\right\}$ on $M_{4}$.

Thus we obtain the following expression for the truncated version $\mathscr{W}_{n}^{T}$ of the holomorphic extension $\mathscr{W}_{n}$ of $S_{n}$, associated with $\mathscr{W}_{n}$ in a similar manner as in Lemma 4.1.

$$
\begin{aligned}
& \mathscr{W}_{n}^{T}=\sum_{\substack{m=0 \\
m \text { even }}}^{n}\left(\begin{array}{l}
n \\
m
\end{array}\right) \frac{1}{m+1} \int_{\mathbf{H} \backslash 0} \alpha_{0}^{n-m}|\vec{\alpha}|^{m} v(d \alpha) \\
& \times \operatorname{Sym} \overbrace{(\widetilde{d} \otimes \ldots \otimes \tilde{d} \otimes}^{n-m} \overbrace{\left.\tilde{D}_{M} \otimes \ldots \otimes \widetilde{D}_{M}\right)}^{m / 2} G_{n} .
\end{aligned}
$$

Taking into account the relation

$$
\begin{aligned}
& \sum_{i=1}^{3}\left(e^{0} \wedge e^{i}-\sqrt{-1} *_{M}\left(e^{0} \wedge e^{i}\right), f\right)_{M}^{2} \\
& \quad=-\frac{1}{2}\left(f-\sqrt{-1} *_{M} f, f-\sqrt{-1} *_{M} f\right)_{M}, \quad f \in \Lambda^{2} \mathbf{R}^{4},
\end{aligned}
$$

we introduce a linear partial differential operator

$$
D^{M}: \Gamma\left(T M_{4}\right) \otimes \Gamma\left(T M_{4}\right) \rightarrow C^{\infty}\left(M_{4}\right) \otimes C^{\infty}\left(M_{4}\right)
$$

by

$$
\begin{aligned}
D^{M}\left(u_{1} \otimes u_{2}\right)\left(x_{1}, x_{2}\right)= & \frac{1}{2}\left(d \eta_{M}\left(u_{1}\right)\left(x_{1}\right)-\sqrt{-1} *_{M} d \eta_{M}\left(u_{1}\right)\left(x_{1}\right),\right. \\
& \left.d \eta_{M}\left(u_{2}\right)\left(x_{2}\right)-\sqrt{-1} *_{M} d \eta_{M}\left(u_{2}\right)\left(x_{2}\right)\right)_{M},
\end{aligned}
$$

and, in addition, we set

$$
\operatorname{div} u=-\delta_{M} \eta_{M}(u), \quad u \in \Gamma\left(T M_{4}\right) .
$$

We now reach an explicit description of (the truncated Wightman distribution) $W_{n}^{T}$ which is associated with $W_{n}$ as in Lemma 4.1, with the difference that this time we must take care that the order of test functions is preserved,

$$
\begin{aligned}
& W_{n}^{T}\left(u_{1}, \ldots, u_{n}\right)=\sum_{\substack{m=0 \\
m \text { even }}}^{n}\left(\begin{array}{l}
n \\
m
\end{array}\right) \frac{1}{m+1} \int_{\mathbf{H} \backslash 0} \alpha_{0}^{n-m}|\vec{\alpha}|^{m} v(d \alpha) \\
& \times\left\langle\operatorname{Sym}(\overbrace{\operatorname{div} \otimes \ldots \otimes \operatorname{div}}^{n-m} \otimes \overbrace{D^{M} \otimes \ldots \otimes D^{M}}^{m-m}) u_{1} \otimes \ldots \otimes u_{n}, l_{M}^{*} G_{n}\right\rangle, \\
& u_{1}, \ldots, u_{n} \in \mathscr{S}\left(T M_{4}\right) \text {. }
\end{aligned}
$$


Here $\imath_{M}^{*} G_{n}$ denotes a generalised function defined as boundary value of the holomorphic function $G_{n}$ in the region $T^{n}$.

(4.15) Remark. The exact function space is not really specified so far. The notation $\mathscr{S}\left(T M_{4}\right)$ is used merely for the sake of convenience. We intend to postpone analytical estimates concerning the Fourier-Laplace transformation till our succeeding paper.

Let us now turn to the point left open above: existence of a holomorphic extension $G_{n}$ of $g^{(n)}$. The key step for this is to represent $g^{(n)}$ as the Fourier-Laplace transform of a distribution with appropriate support.

We fix coordinate systems. By choosing an orthogonal frame $\left\{e_{0}, e_{1}, e_{2}, e_{3}\right\}$ on $M_{4}$, with $e_{0}$ in the forward cone, we introduce a coordinate system $\left\{x^{0}, x^{1}, x^{2}, x^{3}\right\}$ in the affine space $M_{4}$. Let $\left\{z^{0}, z^{1}, z^{2}, z^{3}\right\}$ be the complex coordinate system in $M_{4}^{\mathbf{C}}$ with $\imath_{M}^{*} z^{i}=x^{i}, 0 \leqq i \leqq 3$. It is natural to consider a coordinate system $\left\{y^{0}, y^{1}, y^{2}, y^{3}\right\}$ satisfying $\imath_{E}^{*} z^{0}=\sqrt{-1} y^{0}, l_{E}^{*} z^{i}=y^{i}, 1 \leqq i \leqq 3$, on $E_{4}$. The integral representation

$$
g(y) \equiv \frac{1}{4 \pi^{2}|y|^{2}}=\frac{1}{(2 \pi)^{3}} \int_{\mathbf{R}^{3}} e^{-|\vec{p}|\left|y^{0}\right|-\sqrt{-1} \vec{p} \cdot \vec{y}} \frac{d \vec{p}}{2|\vec{p}|}
$$

is our starting point. To avoid notational complication, we first consider a 1-dimensional analogue of the continuation problem. If $\zeta_{1}, \ldots, \zeta_{n}$ are complex numbers with positive real parts which are fixed for the moment, then, after a bit of calculation, we have

$$
\begin{aligned}
& \int_{-\infty}^{\infty} \prod_{i=1}^{n} e^{-\zeta_{i}\left|t-t_{i}\right|} d t=\frac{1}{\zeta_{1}+\ldots+\zeta_{n}} e^{-\zeta_{2}\left(t_{2}-t_{1}\right)} \ldots e^{-\zeta_{n}\left(t_{n}-t_{1}\right)} \\
& \quad+\sum_{j=1}^{n-1} \prod_{i=1}^{j-1} e^{-\zeta_{i}\left(t_{j}-t_{i}\right)\left(t_{j+1}-t_{j}\right)} \\
& \quad \times \int_{0}^{1} e^{-\left\{\left(\zeta_{1}+\ldots+\zeta_{j}\right) s+\left(\zeta_{j+1}+\ldots+\zeta_{n}\right)(1-s)\right\}\left(t_{j+1}-t_{j}\right)} d s \prod_{i=j+2}^{n} e^{-\zeta_{i}\left(t_{i}-t_{j+1}\right)} \\
& \quad+\frac{1}{\zeta_{1}+\ldots+\zeta_{n}} e^{-\zeta_{1}\left(t_{n}-t_{1}\right)} \ldots e^{-\zeta_{n-1}\left(t_{n}-t_{n-1}\right)}, \quad t_{1}<t_{2}<\ldots<t_{n} .
\end{aligned}
$$

Keeping this formula in mind, we introduce several maps and measures to deal with our 4-dimensional case. According to the space-time decomposition $\mathbf{R} e_{0} \oplus\left\{e_{0}\right\}_{M}^{\perp}$, we write $p_{0}$ (respectively $\vec{p}$ ) for the time (respectively space) component of $p \in \operatorname{Hom}\left(\mathbf{R}^{4}, \mathbf{R}\right)$. We denote the forward light cone in the momentum space by $V_{0}^{*+}$, i.e.

$$
V_{0}^{*+}:=\left\{p \in \operatorname{Hom}\left(\mathbf{R}^{4}, \mathbf{R}\right): Q(p)=0,\left\langle p, e_{0}\right\rangle>0\right\},
$$

and define, for $i=0,1, \ldots, n$, maps

$$
\begin{gathered}
P^{(n, 0)}:\left(V_{0}^{*+}\right)^{n-1} \ni \mathbf{p} \mapsto-\left(p^{(1)}+\ldots+p^{(n-1)}\right), \\
P^{(n, i)}:\left(V_{0}^{*+}\right)^{n-1} \ni \mathbf{p} \mapsto p^{(1)}+\ldots+p^{(i-1)}-\left(p^{(i)}+\ldots+p^{(n-1)}\right), \\
P^{(n, n)}:\left(V_{0}^{*+}\right)^{n-1} \ni \mathbf{p} \mapsto p^{(1)}+\ldots+p^{(n-1)} .
\end{gathered}
$$


We shall also consider the maps $K_{i}^{n}, i=0,1, \ldots, n$, defined by

$$
\begin{gathered}
K_{0}^{n}:\left(V_{0}^{*+}\right)^{n-1} \ni \mathbf{p} \mapsto\left(P^{(n, 0)}(\mathbf{p}), p^{(1)}, \ldots, p^{(n-1)}\right) \in \operatorname{Hom}\left(\mathbf{R}^{4}, \mathbf{R}\right)^{n}, \\
K_{i}^{n}:\left(V_{0}^{*+}\right)^{n-1} \times(0,1) \rightarrow \operatorname{Hom}\left(\mathbf{R}^{4}, \mathbf{R}\right)^{n}, \quad i=1,2, \ldots, n-1 \\
(\mathbf{p}, s) \mapsto\left(-p^{(1)}, \ldots,-p^{(i-1)},(1-s) P^{(n, i)}(\mathbf{p})-s\left(\left\|\vec{P}^{(n, i)}(\mathbf{p})\right\|,-\vec{P}^{(n, i)}(\mathbf{p})\right),\right. \\
\left.s\left(\left\|\vec{P}^{(n, i)}(\mathbf{p})\right\|,-\vec{P}^{(n, i)}(\mathbf{p})\right)+s P^{(n, i)}(\mathbf{p})+p^{(i)}, p^{(i+1)}, \ldots, p^{(n-1)}\right) \\
K_{n}^{n}:\left(V_{0}^{*+}\right)^{n-1} \ni \mathbf{p} \mapsto\left(-p^{(1)}, \ldots,-p^{(n-1)}, P^{(n, n)}(\mathbf{p})\right) \in \operatorname{Hom}\left(\mathbf{R}^{4}, \mathbf{R}\right)^{n} .
\end{gathered}
$$

We observe that

$$
\begin{gathered}
\sum_{l=j}^{n}\left(K_{j}^{n}\right)^{(l)}=p^{(j-1)}+\ldots+p^{(n-1)}, \quad i+2 \leqq j \leqq n \\
\sum_{l=i+1}^{n}\left(K_{i}^{n}\right)^{(l)}= \\
\quad s\left(p^{(1)}+\ldots+p^{(i-1)}+\left(\left\|\vec{P}^{(n, i)}(\mathbf{p})\right\|,-\vec{P}^{(n, i)}(\mathbf{p})\right)\right) \\
+(1-s)\left(p^{(i)}+\ldots+p^{(n-1)}\right), \\
\sum_{l=j}^{n}\left(K_{i}^{n}\right)^{(l)}=p^{(1)}+\ldots+p^{(j-1)}, \quad 1 \leqq j \leqq i
\end{gathered}
$$

Since the closed forward cone

$$
\left(V^{+}\right)^{*}:=\left\{k \in \operatorname{Hom}\left(\mathbf{R}^{4}, \mathbf{R}\right):\langle k, x\rangle \geqq 0 \forall x \in V^{+}\right\}
$$

is convex, the image set of the map $K_{i}^{n}$ satisfies Wightman's spectrum condition ([34]) for each $i=0,1, \ldots, n$.

Let $\mu$ be the Lorentz invariant measure $d \vec{p} / 2|\vec{p}|$ on $V_{0}^{*+}$, and denote by $l$ the Lebesgue measure on $(0,1)$. Using the notation $K \circ \mu \equiv \mu \circ K^{-1}$ for the image of a measure $\mu$ under a map $K$, we introduce measures $M_{i}^{n}$ on $\operatorname{Hom}\left(\mathbf{R}^{4}, \mathbf{R}\right)^{n}$ corresponding to the maps $K_{i}^{n}$ as follows:

$$
\begin{gathered}
M_{0}^{n}(d \mathbf{k})=\frac{1}{2\left\|\vec{k}^{(1)}\right\|\left(\left\|\vec{k}^{(1)}\right\|-k_{0}^{(1)}\right)} K_{0}^{n} \circ \mu^{\otimes(n-1)}(d \mathbf{k}), \\
M_{i}^{n}(d \mathbf{k})=\frac{1}{2\left\|\vec{k}^{(i)}\right\|} K_{i}^{n} \circ\left(\mu^{\otimes(n-1)} \otimes l\right)(d \mathbf{k}), \quad i=1,2, \ldots, n-1, \\
M_{n}^{n}(d \mathbf{k})=\frac{1}{2\left\|\vec{k}^{(n)}\right\|\left(\left\|\vec{k}^{(n)}\right\|+k_{0}^{(n)}\right)} K_{n}^{n} \circ \mu^{\otimes(n-1)}(d \mathbf{k}) .
\end{gathered}
$$

Let

$$
e(\mathbf{z}, \mathbf{k})=(2 \pi)^{-4(n-1)} \exp \left[\sqrt{-1} \sum_{i=1}^{n}\left\langle k^{(i)}, z_{i}\right\rangle\right]
$$

and

$$
\partial_{(i)}^{0}=\frac{\partial}{\partial k_{0}^{(i)}}
$$


Using (4.16) it is not hard to show that the Fourier transform

$$
\begin{aligned}
G_{n}(\mathbf{z}): & =\left\langle e(\mathbf{z}, \cdot), M_{0}^{n}+\sum_{i=1}^{n-1}\left(\partial_{(i+1)}^{0}-\partial_{(i)}^{0}\right) M_{i}^{n}+M_{n}^{n}\right\rangle \\
& =\left\langle e(\mathbf{z}, \cdot), M_{0}^{n}-\sum_{i=1}^{n-1} \sqrt{-1}\left(z_{(i+1)}^{0}-z_{(i)}^{0}\right) M_{i}^{n}+M_{n}^{n}\right\rangle, \mathbf{z} \in T^{n},
\end{aligned}
$$

is a holomorphic extension of $g^{(n)} \mid\left(E_{4}\right)_{<}^{n}$.

Since $g^{(n)}$ is Euclidean invariant and symmetric, $G_{n}$ has an analytic continuation to the permuted extended forward tube, following known procedures (Jost [29], Streater-Wightman [35]) of axiomatic quantum field theory. This is also true for $n=2$, assuming that we take the finite part, i.e. $g^{(2)}$ is defined by Eq. (4.13).

(4.21) Theorem. Let the functions $g_{n}$ on $\left(E_{4}\right)_{\neq}^{n}, n \geqq 2$, be given by (4.12) for $n \geqq 3$ and by (4.13) for $n=2$. Then each $g^{(n)}$ has a holomorphic extension $G_{n}$ defined on the permuted extended forward tube $T_{\text {p.e. }}^{n}$. Denote the boundary value of the holomorphic function $G_{n}$ in the subregion $T^{n}$, the forward tube, by $\imath_{M}^{*} G_{n}$, and set

$$
\begin{aligned}
W_{2}\left(u_{1}, u_{2}\right)= & W_{2}^{T}\left(u_{1}, u_{2}\right)=\left\langle c_{0} \operatorname{div} u_{1} \otimes \operatorname{div} u_{2}+c D^{M}\left(u_{1} \otimes u_{2}\right), l_{M}^{*} G_{2}\right\rangle, \\
& u_{1}, u_{2} \in \mathscr{S}\left(T M_{4}\right),
\end{aligned}
$$

where

$$
c_{0}=\sigma_{0}+\int_{\mathbf{H}\{\{0\}} \alpha_{0}^{2} v(d \alpha), \quad c=\sigma+\frac{1}{3} \int_{\mathbf{H}\{\{0\}}|\alpha|^{2} v(d \alpha) .
$$

For $n \geqq 3$, set

$$
\begin{aligned}
& W_{n}^{T}\left(u_{1}, \ldots, u_{n}\right)=\sum_{\substack{m=0 \\
m \text { even }}}^{n}\left(\begin{array}{c}
n \\
m
\end{array}\right) \frac{1}{m+1} \int_{\mathbf{H} \backslash 0} \alpha_{0}^{n-m}|\vec{\alpha}|^{m} v(d \alpha) \\
& \times\left\langle\operatorname{Sym}(\overbrace{\operatorname{div} \otimes \ldots \otimes \operatorname{div}}^{n-m} \otimes \overbrace{D^{M} \otimes \ldots \otimes D^{M}}^{m / 2}) u_{1} \otimes \ldots \otimes u_{n}, l_{M}^{*} G_{n}\right\rangle, \\
& u_{1}, \ldots, u_{n} \in \mathscr{S}\left(T M_{4}\right) \text {. }
\end{aligned}
$$

Then $\left\{W_{n}^{T}\right\}$ are the generalised functions (hyperfunctions) obtained by holomorphically continuing the cumulants of the random field A of Theorem 3.9. They satisfy the postulates on invariance, spectral property, and locality in axiomatic quantum field theory.

For $n=2$, the holomorphic extension $\mathscr{W}_{2}$ is represented by elementary functions with domain of holomorphy $\left\{\mathbf{z} \in\left(M_{4}^{\mathbf{C}}\right)^{2}: Q\left(z_{1}-z_{2}\right) \neq 0\right\}$ as follows:

$$
\begin{aligned}
\mathscr{W}_{2}\left(z_{1}, z_{2}\right)= & \left\{\left(c+c_{0}\right) \frac{\eta_{i j}}{8 \pi^{2} Q\left(z_{1}-z_{2}\right)}\right. \\
& \left.-\left(c_{0}-c\right) \sum_{i^{\prime} j^{\prime}} \eta_{i i^{\prime}} \eta_{j j^{\prime}} \frac{\left(z_{1}^{i^{\prime}}-z_{2}^{i^{\prime}}\right)\left(z_{1}^{j^{\prime}}-z_{2}^{j^{\prime}}\right)}{4 \pi^{2} Q\left(z_{1}-z_{2}\right)^{2}}\right\} d z_{1}^{i} \otimes d z_{2}^{j},
\end{aligned}
$$

and $W_{2}=i_{M}^{*} \mathscr{W}_{2}$. Here $\left(\eta_{i j}\right)$ denotes the Minkowski metric and $Q$ its associated quadratic form.

Proof. It remains to prove (4.22) and (4.24), and the point is that we can easily see the domain of holomorphy for $\mathscr{W}_{2}$. To derive (4.22) we need to take the finite part. 
This can be achieved by regularising $g$ to $g_{\varepsilon}$, say, so that the latter function is meromorphic with respect to $\varepsilon$ and let $\varepsilon \downarrow 0$. (See e.g. Hörmander [25], Chap. 3.) Then we obtain (4.14) for $n=2$ with $g^{(2)}$ defined as in (4.13), and therefore we have (4.22).

On the other hand we have from the identities (3.13) and (4.9) that

$$
\begin{aligned}
S_{2}(u, u)= & U_{2}(\langle u, A\rangle\langle u, A\rangle)=c \int_{E_{4}}|g * \partial u(x)|^{2} d x+\left(c_{0}-c\right) \int_{E_{4}}(\operatorname{Re} g * \partial u(x))^{2} d x \\
= & c \int_{\left(E_{4}\right)^{2}} g\left(y_{1}-y_{2}\right)\left(u\left(y_{1}\right), u\left(y_{2}\right)\right)_{E} d \mathbf{y} \\
& +\left(c_{0}-c\right) \int_{E_{4}}\left\{\int_{\left(E_{4}\right)^{2}}\left\langle d g(x-\cdot)\left(y_{1}\right), u\left(y_{1}\right)\right\rangle\left\langle d g(x-\cdot)\left(y_{2}\right), u\left(y_{2}\right)\right\rangle d \mathbf{y}\right\} d x \\
= & \int_{\left(E_{4}\right)^{2}}\left\{c g\left(y_{1}-y_{2}\right)\left(u\left(y_{1}\right), u\left(y_{2}\right)\right)_{E}\right. \\
& +\left(c_{0}-c\right)\left\langle d \otimes d g^{(2)}(\mathbf{y}), u \otimes u(\mathbf{y})\right\rangle d \mathbf{y} .
\end{aligned}
$$

Here we have again used the regularisation method for the divergent integral.

We set $G_{2}(\mathbf{z})=-\log Q\left(z_{1}-z_{2}\right) /\left(16 \pi^{2}\right), \quad \mathbf{z} \in T^{2}$. Then $l_{E}^{*} G_{2}=g^{(2)}$ on $\left(E_{4}\right)_{<}^{2}$. Carrying out the differentiation we have

$$
\begin{aligned}
d \otimes d G_{2}(\mathbf{z})= & \frac{\eta_{i j}}{8 \pi^{2} Q\left(z_{1}-z_{2}\right)} d z_{1}^{i} \otimes d z_{2}^{j} \\
& -\frac{1}{4 \pi^{2} Q\left(z_{1}-z_{2}\right)^{2}} \sum_{\substack{0 \leqq i, i^{\prime} \leqq 3 \\
0 \leqq j, j^{\prime} \leqq 3}} \eta_{i i^{\prime}} \eta_{j j^{\prime}}\left(z_{1}-z_{2}\right)^{i^{\prime}}\left(z_{1}-z_{2}\right)^{j^{\prime}} d z_{1}^{i} \otimes d z_{2}^{j},
\end{aligned}
$$

hence the formula (4.24). Finally this explicit formula (4.24) shows that the domain of holomorphy of $\mathscr{W}_{2}$ is $\left\{z \in\left(M_{4}^{\mathbf{C}}\right)^{2}: Q\left(z_{1}-z_{2}\right) \neq 0\right\}$.

(4.26) Remarks. (i) The special form for $n=2$ shows, again (see Sect. 3), that the Gaussian case is exceptional. Another expression for this exceptionality is that the functions $\mathscr{W}_{2}$ are single-valued. Due to this fact, we obtain the same boundary values from the tube $T^{n}$ with base $V^{+}[\operatorname{see}(4.7)]$ and the tube with base $-V^{+}$. In other words, the cases $z_{i+1}-z_{i} \in \mathbf{R}^{4} \pm J V^{+}$give the same result. It follows that in the Gaussian case the field commutes for $Q\left(x_{1}-x_{2}\right) \neq 0$ in the sense that then

$$
\left(\Omega, A\left(x_{1}\right) A\left(x_{2}\right) \Omega\right)=\left(\Omega, A\left(x_{2}\right) A\left(x_{1}\right) \Omega\right) .
$$

(ii) One can regularise and replace $g$ in the formulae above by

$$
g_{\varepsilon}(y)=\frac{1}{(2 \pi)^{3}} \int_{\mathbf{R}^{3}} e^{-\omega_{\varepsilon}(\vec{p})\left|y^{0}\right|-\sqrt{-1} \vec{p} \cdot \vec{y}} \frac{d \vec{p}}{2 \omega_{\varepsilon}(\vec{p})},
$$

where $\omega_{\varepsilon}(\vec{p})=\left(|\vec{p}|^{2}+\varepsilon^{2}\right)^{1 / 2}$. Replacing $V_{0}^{*+}$ in (4.17) by the forward mass hyperboloid where $Q(p)=-\varepsilon^{2}$, and performing corresponding modifications in (4.18) etc., it is not difficult to see that the corresponding Wightman functions $W_{n, \varepsilon}^{T}$ are in fact tempered distributions for $\varepsilon>0$.

(iii) In (4.25) the parameters $c_{0}, c \geqq 0$ enter. $c / c_{0}$ is a kind of gauge parameter. In the Gaussian case $c_{0}=c$ is the Feynman gauge and $c_{0}=0$ is the Landau gauge.

(iv) In axiomatic approaches to relativistic quantum fields other requirements are made in addition to the above verified properties of invariance, spectrum and 
locality, in particular the positivity property of Wightman functions. We shall postpone the discussion of these properties to later work, since it is connected with the above mentioned question of the proper choice of test function space. Our fields being of gauge type, this point is related to well known problems of gauge field theories, see e.g. Jakobczyk and Strocchi [27].

Acknowledgements. This work was supported by the Mathematical Department, Royal Inst. Technology, Stockholm, and by grants from the Royal Swedish Academy of Sciences, the Swedish Natural Science Research Council, NFR, the Swedish National Board for Technical Development, STU-F, and the Sonderforschungsbereich 237 (Bochum-Essen-Düsseldorf).

Many of the basic ideas in this and the related previous work are due to Raphael HøeghKrohn. We dedicate this article to his memory.

\section{References}

0. Albeverio, S.: Some new developments concerning Dirichlet Forms, Markov fields and quantum fields, pp. 250-259. In: Proc. IX IAMP Congress on Math. Physics, Swansea 1988. Simon, B., Truman, A., Davies, I.M., Hilger, A., Bristol (eds.) (1989)

1. Albeverio, S., Blanchard, P., Combe, P., Høegh-Krohn, R., Sirugue, M.: Local relativistic invariant flows for quantum fields. Commun. Math. Phys. 90, 329-351 (1983)

2. Albeverio, S., Høegh-Krohn, R.: Diffusion fields, quantum fields, and fields with values in groups, pp. 1-98. Pinsky, M. (ed.). Stochastic Analysis and Applications. New York: M. Dekker 1984

3. Albeverio, S., Høegh-Krohn, R.: Euclidean Markov fields and relativistic quantum fields from stochastic partial differential equations in four dimensions. Phys. Lett. B 177, 175-179 (1986)

4. Albeverio, S., Høegh-Krohn, R.: Quaternionic non-abelian relativistic quantum fields in four space-time dimensions. Phys. Lett. B 189, 329-336 (1987)

5. Albeverio, S., Høegh-Krohn, R.: Construction of interacting local relativistic quantum fields in four space-time dimensions. Phys. Lett. B 200, 108-114 (1988), with erratum in ibid. 202, p. 621 (1988)

6. Albeverio, S., Fenstad, J.E., Høegh-Krohn, R., Lindstrøm, T.: Nonstandard methods in stochastic analysis and mathematical physics. New York: Academic Press 1986

7. Albeverio, S., Høegh-Krohn, R., Holden, H.: Markov cosurfaces and gauge fields. Acta Phys. Austr. [Suppl.] XXVI, 211-231 (1984)

8. Albeverio, S., Høegh-Krohn, R., Holden, H.: Stochastic multiplicative measures, generalised Markov semigroups and group-valued stochastic processes and fields. J. Funct. Anal. 78, 154-184 (1987)

9. Albeverio, S., Høegh-Krohn, R., Holden, H., Kolsrud, T.: Representation and construction of multiplicative noise. J. Funct. Anal. 87, 250-272 (1989)

10. Albeverio, S., Høegh-Krohn, R., Holden, H., Kolsrud, T.: A covariant Feynman-Kac formula for unitary bundles over Euclidean space, pp. 1-12. In: Proc. Conf. "Stochastic Partial Differential Equations II", Trento Feb. 1988. Da Prato, G., Tubaro, L. (eds.). Lecture Notes in Math., vol. 1390. Berlin, Heidelberg, New York: Springer 1989

11. Albeverio, S., Høegh-Krohn, R., Holden, H., Kolsrud, T.: Construction of quantised Higgslike fields in two dimensions. Phys. Lett. B 222, 263-268 (1989)

12. Albeverio, S., Høegh-Krohn, R., Iwata, K.: Covariant markovian random fields in four spacetime dimensions with nonlinear electromagnetic interaction. In: Applications of self-adjoint extensions in quantum physics. Proc. Dubna Conference 1987. Exner, P., Seba, P. (eds.). Lecture Notes in Phys., vol. 324. Berlin, Heidelberg, New York: Springer 1989

13. Albeverio, S., Høegh-Krohn, R., Zegarlinski, B.: Uniqueness and global Markov property for Euclidean fields. The case of general polynomial interactions. Commun. Math. Phys. 123, 377-424 (1989) 
14. Balaban, T.: Large field renormalization. I. The basic step of the $\mathbf{R}$ operation. Commun. Math. Phys. 122, 175-202 (1989)

15. Barut, A.O., Raczka, R.: Theory of group representations and applications, 2nd ed. Singapore: World Scientific 1986

16. Berg, C., Forst, G.: Potential theory on locally compact abelian groups. Berlin, Heidelberg, New York: Springer 1975

17. Born, M., Infeld, L.: Foundations of the new field theory. Proc. R. Soc. A 144, 425-451 (1934)

18. Gawedzki, J.K., Kupiainen, A.: Massless lattice $\varphi_{4}^{4}$ theory: Rigourous control of a renormalizable asymptotically free model. Commun. Math. Phys. 99, 197-252 (1985)

19. Gelfand, I.M., Vilenkin, N.Ya.: Generalized functions, vol. IV. New York: Academic Press 1964

20. Glimm, J., Jaffe, A.: Quantum Physics, second ed., Berlin, Heidelberg, New York: Springer 1987

21. Gross, L., King, C., Sengupta, A.: Two dimensional Yang-Mills theory via stochastic differential equations. Ann. Phys. 194, 65-112 (1989)

22. Guerra, F., Loffredo, M.I.: Stochastic equations for the Maxwell field. Lett. Nuovo Cim. 27, 41-45 (1980)

23. Guerra, F., Ruggiero, P.: A new interpretation of the Euclidean-Markov field in the framework of physical Minkowski space-time. Phys. Rev. Lett. 31, 1022-1025 (1973)

24. Haba, Z.: Stochastic equations for some Euclidean fields, pp. 315-328. In: Proc. "Stochastic Processes in Classical and Quantum Systems", Ascona. Albeverio, S., Casati, G., Merlini, D. (eds.). Lecture Notes Phys., vol. 262. Berlin, Heidelberg, New York: Springer 1986

25. Hörmander, L.: The analysis of linear partial differential operators, vol. I. Berlin, Heidelberg, New York: Springer 1983

26. Itô, K.: Foundations of stochastic differential equations in infinite dimensional spaces. CBMS-NSF Regional Conf. Series in Appl. Math. Vol. 47. SIAM 1984

27. Jakobczyk, L., Strocchi, F.: Euclidean formulation of quantum field theory without positivity. Commun. Math. Phys. 119, 529-541 (1988)

28. Jona-Lasinio, G., Mitter, P.K.: On the stochastic quantization of field theory. Commun. Math. Phys. 101, 409-436 (1985)

29. Jost, R.: The general theory of quantised fields. Providence, RI: Am. Math. Soc. 1965

30. Kobayashi, S., Nomizu, K.: Foundations of differential geometry, vol. I-II. New York: Wiley 1963, 1969

31. Kusuoka, S.: Markov fields and local operators. J. Fac. Sci. Univ. Tokyo 1A, 26, 199-212 (1979)

32. Osipov, E.P.: Euclidean Markov fields from stochastic partial differential equations in eightdimensional space. Novosibirsk preprint 1987

33. Royden, H.L.: Real Analysis, 2nd ed. MacMillan 1968

34. Simon, B.: The $P(\varphi)_{2}$ Euclidean (quantum) field theory. Princeton, NJ: Princeton University Press 1974

35. Streater, R.F., Wightman, A.S.: PCT, spin, statistics and all that. New York: Benjamin 1964

36. Sudbery, A.: Quaternionic analysis. Math. Proc. Cambridge Phil. Soc. 85, 199-225 (1979)

37. Surgailis, D.: On the Markov property of a class of linear infinitely divisible fields. Z. Warsch. Verw. Geb. 49, 293-311 (1979)

38. Surgailis, D.: On covariant stochastic differential equations and Markov property of their solutions. Preprint, Univ. di Roma, Fisica, 1979

39. Yamasaki, Y.: Measures on infinite dimensional spaces. Singapore: World Scientific 1985

Communicated by K. Gawedzki

Received June 18, 1989 